\title{
Geometrically Non-Linear Modeling of the Portevin-Le Chatelier Effect
}

\author{
T. Böhlke1 , G. Bondár ${ }^{1}$, Y. Estrin², M.A. Lebyodkin ${ }^{3}$ \\ ${ }^{1}$ : Kontinuumsmechanik im Maschinenbau, Institut für Technische Mechanik \\ Karlsruhe Institute of Technology, Postfach 6980, D-76128 Karlsruhe \\ 2: ARC Centre of Excellence for Design in Light Metals, Department of Materials Engineering, Monash University, \\ Clayton,VIC 3800, Australia and CSIRO Division of Manufacturing and Materials Technology, Clayton, Vic. 3168, \\ Australia \\ ${ }^{3}$ : Laboratoire de Physique et Mécanique des Matériaux, UMR CNRS N ${ }^{\circ} 7554$, \\ Université Paul Verlaine-Metz, Ile du Saulcy, 57045 Metz Cedex, France
}

ABSTRACT. In this work we investigate the plastic instabilities associated with the Portevin-Le Chatelier (PLC) effect in Al alloy 2024. A semiphenomenological approach is taken. A simple geometrically non-linear elastic-viscoplastic constitutive model is proposed for simulation of material response under various applied strain rates. Using the model we determine numerically the relation between the critical strain for the onset of discontinuous yielding and the applied strain rate. The results obtained are in very good quantitative agreement with the available experimental data and cover both the normal and the inverse behavior of the critical strain. The simulations are performed using non-linear finite element method. Additional verification of the proposed constitutive framework was carried out using statistical analysis of the simulated stress-time series. A transition from a non-linear chaotic regime to self-organized critical behaviour of the localized strain bands were predicted in terms of the temporal two-point correlation function of the stress-time series. Finally we investigated the influence of different factors, such as the geometry of the specimen, its orientation with respect to the rolling direction and loading conditions (strain rate), on the type of PLC instabilities and the critical conditions for their onset.

KEYWORDS: plastic instability, dynamic strain aging, non-linear finite element method, normal and inverse behaviour, jerky flow 


\section{Contents}

1 Introduction 3

2 Experimental data $\quad 5$

$\begin{array}{lll}3 & \text { Constitutive equations } & 7\end{array}$

3.1 Motivation . . . . . . . . . . . . . . . . . 8

3.2 Three-dimensional geometrically linear model . . . . . . . . . . . . 10

3.3 Three-dimensional geometrically non-linear model . . . . . . . . . . 12

4 Numerical implementation $\quad 13$

4.1 Numerical time integration scheme . . . . . . . . . . . . . . . . 13

4.2 Parameter identification . . . . . . . . . . . . . . . 15

5 Results and discussion $\quad 16$

5.1 Statistical analysis . . . . . . . . . . . . . . . . . . . 16

5.2 Band characteristics in tension and compression . . . . . . . . . . . . 19

5.3 Rôle of the dimensionality of the strain state . . . . . . . . . . . . 22

5.4 Deep drawing of a metal sheet . . . . . . . . . . . . . . . 23

6 Summary and Conclusions 24 


\section{Introduction}

Dynamic interaction between mobile dislocations and solute atoms results for many industrially relevant dilute metallic alloys (notably Al-, Cu- or Fe-based ones) in negative strain rate sensitivity of the flow stress. In a certain range of strain rates and temperatures, this leads to destabilisation of the uniformity of plastic flow in these materials (Neuhäuser, 1990; Estrin and Kubin, 1995). The instability takes the form of propagating localised deformation bands and concomitant repetitive stress drops ('discontinuous yielding'). It is generally accepted that the micromechanical mechanism underlying this destabilising effect is the dynamic aging of dislocations by diffusing solute atoms (Cottrell, 1953b,a; Sleeswyk, 1958; McCormick, 1988; van den Beukel and Kocks, 1982; Schlipf, 1994). In uniaxial strain-controlled tensile deformation tests (Estrin, 1987; Estrin and Kubin, 1995; Zaiser and Hähner, 1997; Ziegenbein et al., 2001; Dierke et al., 2007) dynamic strain aging (the Portevin-Le Chatelier effect) manifests itself through serrations on the stress-strain curve (Portevin and Le Châtelier, 1923; Bell, 1973). From a technological perspective, this effect reduces the ductility of the material and corrupts the surface quality of semi-products (appearance of surface markings and waviness). The Portevin-Le Chatelier (PLC) effect reduces the formability of the material in deep-drawing processes, e.g. in sheet-metal forming. Depending on the spatiotemporal organisation of the deformation bands, different types of PLC instabilities can occur. These are categorised as PLC instabilities of types A, B and C. A more detailed taxonomy of PLC bands has also been used (Pink and Greenberg, 1982). PLC bands of type $C$ appear in the sample almost ${ }^{1}$ at random without propagating (stochastic nucleation), type $B$ bands exhibit an oscillatory or intermittent propagation along the specimen's tensile axis in a kind of relay-race manner, and, finally, type $A$ bands propagate continuously, as solitary plastic waves, along the tensile axes. During the uniaxial tensile tests conducted for the purposes of the present project on aluminium alloy 2024, we observed - dependent on the strain rate - the occurrence of localised deformation bands (PLC instabilities) of all three types. At small strain rates, the plastic flow was heavily discontinuous with bursts of plastic activity without correlation in time and space, and bands of type $\mathrm{C}$ emerged. At large strain rates the quasi-periodic stress drops observed were associated with the nucleation of PLC bands of type A, which then propagated in the axial direction in a way similar to the propagation of the Lüders bands (Lüders, 1860). In addition to the characterisation of the PLC effect in terms of the type of the deformation bands, the critical equivalent strain after which

\footnotetext{
${ }^{1}$ In a recent study (Lebyodkin and Lebedkina, 2008) it was shown that these appear as a result of weakly correlated nucleation events.
} 
strain localisation sets in is commonly used as an important practical characteristic of PLC instability. The critical strain depends on the strain rate and temperature. A common case reported in the literature is that of the critical strain increasing with increasing strain rate. This kind of behaviour, referred to as 'normal', is typical of many dilute alloys (Estrin and Kubin, 1995). The 'inverse' behaviour characterised by a decrease of the critical strain with increased strain rate was also reported occasionally (Kubin and Estrin, 1992a; Estrin and Kubin, 1995). While some authors (Brechet and Estrin, 1995) associate the inverse effect with precipitate shearing and re-constitution of precipitate during deformation, others (Hähner et al., 2002) modified the ansatz for the aging time and used a linear stability analysis to trace the inverse behaviour back to the dynamic strain aging kinetics and dislocation dynamics. The latter approach accounts for a nonmonotonic dependence of the critical strain on the strain rate exhibiting a minimum, or a plateau in the critical strain over a range of intermediate strain rates.

Since the experimental identification of the inverse PLC effect and analysis of its spatial aspects pose an enormous experimental challenge, numerical simulations remain the main tool for its study. Over the years, a significant research effort has been devoted to theoretical modelling and numerical simulations of the strain aging effects (Kubin and Estrin, 1990, 1992b; Mesarovic, 1995; Zaiser and Hähner, 1997; Valanis, 2000; Hähner et al., 2002), kinematic characterisation of the PLC bands (McCormick et al., 1993; Hähner, 1993; Dablij and Zeghloul, 1997), and the numerics of this particular type of instabilities (Kubin et al., 1988; McCormick and Ling, 1995; Lebyodkin et al., 1996, 2000). Despite the large number of published works, however, the predictive capabilities of the dynamic strain aging models are not satisfactory, especially concerning the inverse behavior of the alloys. Precise and reliable determination of the critical strain for the onset of plastic instability as well as further quantification of the inverse PLC behaviour are the two major challenges yet to be resolved by the non-linear constitutive modelling and numerical simulations.

In this study we propose a novel geometrically and physically non-linear material model for reliable simulation of the dynamic strain aging, and in particular the inverse behavior of the critical strain for the onset of the PLC effect, in samples of an $\mathrm{Al}$ alloy subjected to three-dimensional loading conditions. Our approach is phenomenological, although it is related to earlier microstructural approaches (Kubin and Estrin, 1990, 1992a; Zhang et al., 2001). The model is elastic-viscoplastic and is based on a single evolution equation for the dynamic strain aging. Exploring the concept of internal variables in a purely mechanical context we formulate the complex hardening response of the material from a phenomenological point of view proposing a simple 
constitutive ansatz based on a non-linear formulation of the strain hardening law (of Voce type) with account for strain rate effects (logarithmic strain rate dependence) and a modified dynamic strain aging (Cottrell and Bilby, 1949; Head and Louat, 1955). The model is gauged for the Al alloy investigated and further utilized for studying the influence of various factors, including the geometry of the specimen, deformation conditions (strain rate control), orientation with respect to the rolling direction etc., on the type of the PLC instabilities and the critical conditions for their onset.

\section{Experimental data}

In the first series of experiments we conducted uniaxial tensile tests with samples of $\mathrm{Al}$ alloy 2024, in the following range of applied strain rates: $\dot{\varepsilon}_{a}=1 \cdot 10^{-5}-1 \cdot 10^{-1} \mathrm{~s}^{-1}$. The samples were annealed at $493^{\circ} \mathrm{C}$ for 40 minutes and quenched in polyalkylene glycol solution. Prior to mechanical tests the specimens were kept in liquid nitrogen. The goal was to determine the instability range and the general features of the deformation response for this diluted alloy. The following nominal applied strain rates were used: $\dot{\varepsilon}_{a}=1 \cdot 10^{-5}, 1 \cdot 10^{-4}, 1 \cdot 10^{-3}, 1 \cdot 10^{-2}, 7 \cdot 10^{-2}, 1 \cdot 10^{-1} \mathrm{~s}^{-1}$. In a second series of experiments, tensile tests were conducted for three groups of samples characterized by different orientation of the tensile axis with respect to the rolling direction $\left(0^{\circ}, 45^{\circ}, 90^{\circ}\right)$. The following applied strain rates were used: $\dot{\varepsilon}_{a}=3 \cdot 10^{-5}, 3 \cdot 10^{-4}, 3 \cdot 10^{-3}, 3 \cdot 10^{-2}, 7 \cdot 10^{-1} \mathrm{~s}^{-1}$. A recorded signal from a standard extensometer provides local information about the strain field within the gauged segment of the sample, and this information cannot be considered as representative because of the heterogeneity of the deformation field, especially when the strain is localized outside of the extensometer span. For this reason the cross-head displacement of the testing machine was used for determining the overall strain in the sample. Disregarding errors at small strains $(<0.2 \%-0.3 \%)$ associated with non-linearity of the testing machine, we present all data in terms of the engineering stresses and strains. (It should also be noted that the elastic component of the total strain corresponds to the elastic response of the tensile specimen + testing machine system.) The experimental stress-strain curves for the mentioned strain rates are shown in Fig. 1 a)-d). For comparison, a triad of curves corresponding to three different orientations and recorded at a particular strain rate are presented in each diagram along with those for $10^{-5} \mathrm{~s}^{-1}$ taken as a reference. The findings can be summarized as follows:

i) The stress-strain curves for $90^{\circ}$ and $45^{\circ}$ are very close; the samples with $0^{\circ}$ of orientation exhibit a higher rate of strain hardening, and the corresponding curves lie above those for the other two orientations. Thus, the orientation of the sample 
with respect to the rolling direction has a significant influence on the stress levels.

ii) For strain rates above $10^{-2} \mathrm{~s}^{-1}$ stress jumps degenerate to undulations on the deformation curve, mainly due to elastic reloading of the sample during a stress drop, which results in smoothening of the saw-tooth profile on $\sigma-\varepsilon$ diagram. The strain at which undulations set in is as high as about $10 \%$ or above, and for strain rates in excess of $10^{-2} \mathrm{~s}^{-1}$, unstable behaviour disappears altogether. It should be noted that the waviness of the part of the stress-strain curve corresponding to unstable plastic flow for strain rates in the upper part of the strain rate range considered makes it difficult to identify the critical strain for the onset of instability accurately enough.

iii) Figure 2 shows the strain rate dependence of the critical strains and stresses in a semi-logarithmic diagram. At small strain rates, $\dot{\varepsilon}_{a}=1 \cdot 10^{-4}-1 \cdot 10^{-3} \mathrm{~s}^{-1}$ the material responds to an increase in the strain rate in an 'inverse' way, i.e. the critical strain decreases with increasing strain rate. In the transition range from type $\mathrm{C}$ to type $\mathrm{B}$ to type $\mathrm{A}$ behaviour $\dot{\varepsilon}_{a}=10^{-3}-10^{-2} \mathrm{~s}^{-1}$ a plateau in the strain rate dependence of $\varepsilon_{c}$ is observed: in this range of conditions, the critical strain is not sensitive to the strain rate. Finally, for $\dot{\varepsilon}_{a}$ above $10^{-2} \mathrm{~s}^{-2}$ in the range of type A PLC bands, a normal behavior characterized by $\varepsilon_{c}$ rising with $\dot{\varepsilon}_{a}$ is observed until the PLC instability disappears altogether. The influence of the specimen orientation with respect to the rolling direction on the critical strain and the type of the PLC bands appears to be of minor importance for the material investigated. The variation of the critical strain with the strain rate in the inverse region is so rapid that in the low strain rate part of the strain rate range considered, at $\dot{\varepsilon}_{a}=$ $10^{-4} \mathrm{~s}^{-1}$, the specimens fail prior to the onset of the PLC instability, and serrations are only seen in the post-failure part of the stress-strain curves.

iv) Finally, no serrations were observed on the stress-strain curves corresponding to the lowest strain rate studied, $\dot{\varepsilon}_{a}=1.0 \cdot 10^{-5} \mathrm{~s}^{-1}$.

The experimental results reported here were used for calibration and verification of the constitutive model presented below. The data provide a reliable and representative information for a broad strain rate range covering both the normal and the inverse PLC effect. 


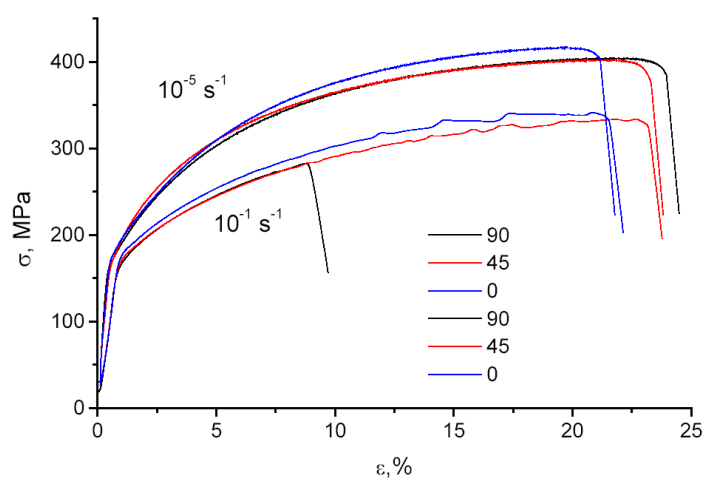

a)

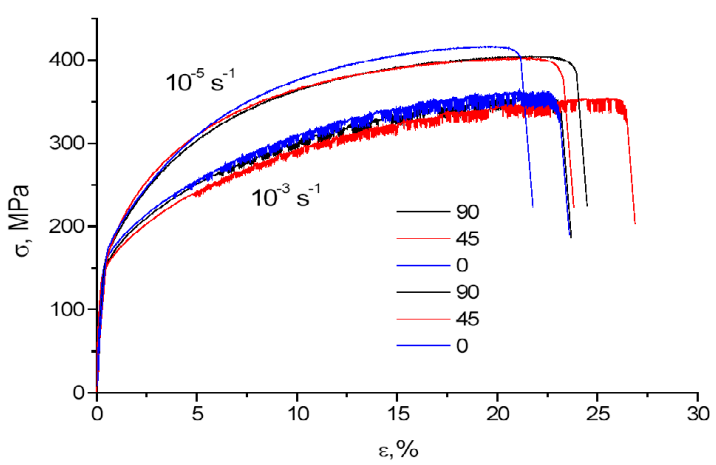

c)

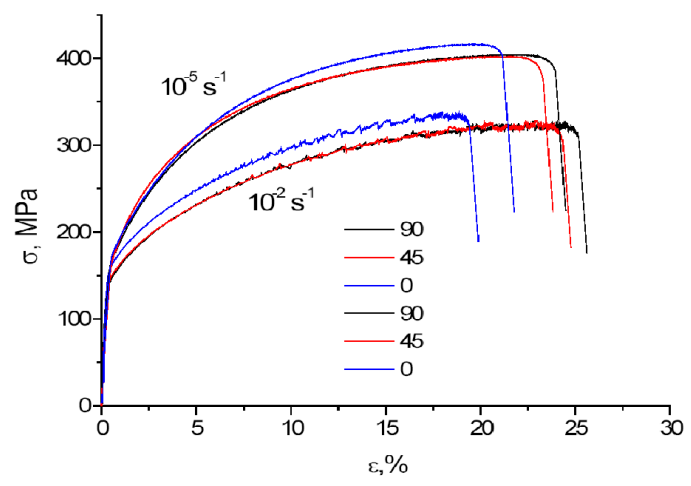

b)

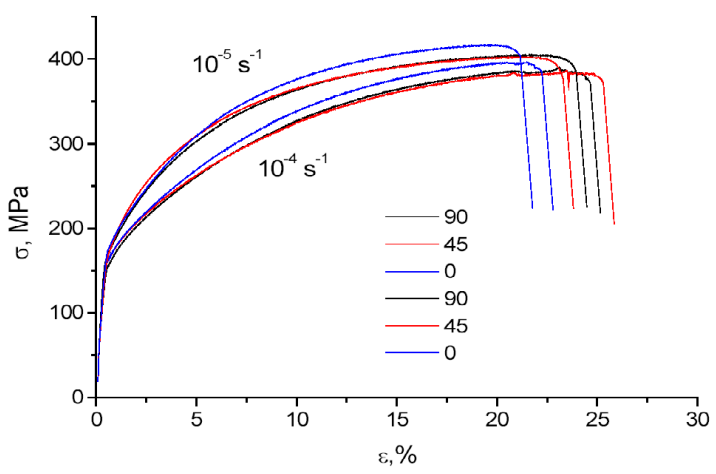

d)

Figure 1: Experimental stress-strain curves for the strain rates: a) $\dot{\varepsilon}_{a}=1 \cdot 10^{-1} \mathrm{~s}^{-1}$; b) $\dot{\varepsilon}_{a}=1 \cdot 10^{-2} \mathrm{~s}^{-1}$; c) $\left.\dot{\varepsilon}_{a}=1 \cdot 10^{-3} \mathrm{~S}^{-1} ; \mathrm{d}\right) \dot{\varepsilon}_{a}=1 \cdot 10^{-4} \mathrm{~S}^{-1}$.

\section{Constitutive equations}

As already mentioned in the Introduction, our principal aim was to develop a constitutive model accounting for both the normal and the inverse behavior under dynamic strain aging conditions. Our research was driven by the fact that the existing models are commonly not capable of accounting for both types of behaviour within a single approach. A brief overview of the situation that gave us the motivation for embarking on the present study is given below. 


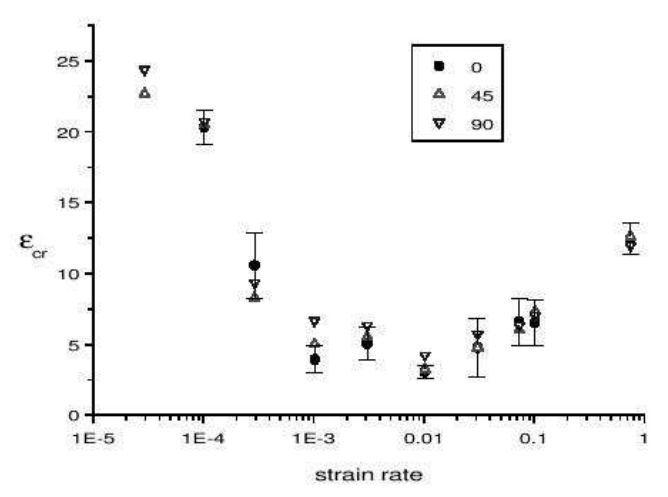

a)

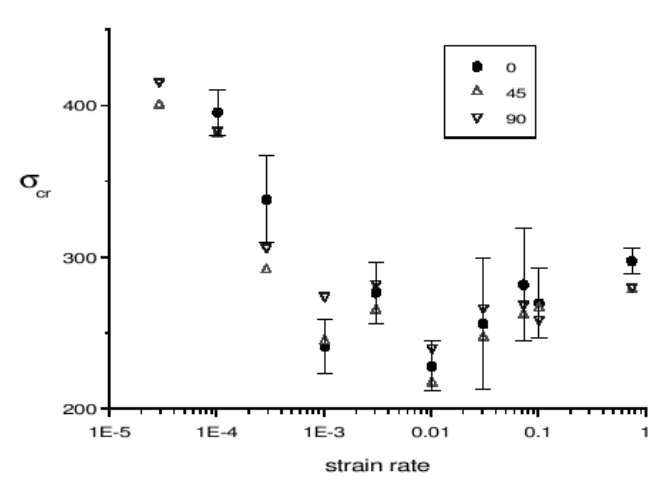

b)

Figure 2: Experimental stability maps: a) critical strain $\varepsilon_{c r}$ vs. strain rate; and b) critical stresses $\sigma_{c r}$ vs. strain rate.

\subsection{Motivation}

Consider a one-dimensional model similar to the one suggested by Penning (1972). The flow stress $\sigma_{F}$ is decomposed in three additive parts

$$
\sigma_{F}=\sigma_{H}\left(\varepsilon_{p}\right)+\sigma_{V}\left(\dot{\varepsilon}_{p}\right)+\sigma_{B}\left(\varepsilon_{p}, t_{a}\right)
$$

with

$$
\sigma_{V}=S_{0} \ln \left(\frac{\dot{\varepsilon}_{p}}{\dot{\varepsilon}_{0}}\right), \quad \sigma_{B}=\sigma_{B 0}\left(1-\exp \left(-\left(\frac{t_{a}}{t_{0}}\right)^{n}\right)\right) .
$$

The stress $\sigma_{H}$ describes the strain hardening. The stress $\sigma_{V}$ accounts for the contribution to the flow stress with positive strain rate sensitivity. Finally, $\sigma_{B}$ takes the dynamic strain aging into account. It is specified by the Cottrell-Bilby relation (Cottrell and Bilby, 1949) modified by Louat to account for the saturation of solute atmospheres around dislocations (Head and Louat, 1955). The characteristic time for solute diffusion is denoted by $t_{0}$ and the maximum value of this contribution to stress, which is reached at saturation, is given by $\sigma_{B 0}$. The 'dynamic aging' time $t_{a}$ can be assumed to be identical with the waiting time a dislocation spends at localized obstacles and is 'aged' by solutes segregating on it through diffusion: $t_{a}=t_{w}$ with $t_{w}=\Omega\left(\varepsilon_{p}\right) / \dot{\varepsilon}_{p}$ (McCormick, 1988). The quantity $\Omega\left(\varepsilon_{p}\right)$ is the elementary plastic strain that would be produced if all mobile dislocations temporarily pinned at localized obstacles (forest dislocations) were released and moved to the next pinned configuration. Obviously, it should depend on $\varepsilon_{p}$ through the densities of mobile and forest dislocations (Estrin and Kubin, 1995; Kubin and Estrin, 1990, 1992a). As a result, the part of the stress 
accounting for the PLC effect is given by

$$
\sigma_{B}=\sigma_{B 0}\left(1-\exp \left(-\left(\frac{\Omega\left(\varepsilon_{p}\right)}{t_{0} \dot{\varepsilon}_{p}}\right)^{n}\right)\right) .
$$

A criterion separating regions of stable and unstable behaviour in the (strain, strain rate) space can be formulated as the condition of vanishing strain rate sensitivity: $S\left(\varepsilon_{p}, \dot{\varepsilon}_{p}\right):=\partial \sigma_{F} / \partial \ln \left(\dot{\varepsilon}_{p}\right)=0$ (total strain $\varepsilon$ fixed!) (Estrin and Kubin, 1995).

This condition can be seen as the lower bound of instability region 2 (see an example for this bound in Ling and McCormick, 1990). Hence, the instability criterion is given by

$$
\alpha-Z \exp (-Z)=0, \quad \alpha \in[0,1 / e)
$$

with $\alpha=S_{0} /\left(\sigma_{B 0} n\right)$ and $Z=\left(\Omega\left(\varepsilon_{p}\right) /\left(t_{0} \dot{\varepsilon}_{p}\right)\right)^{n}$. Depending on $\alpha$, this equation either has no solution, or has two positive solutions for $Z$. Assume that two positive solutions can be found and denote them by $Z_{1,2}>0$. The boundary of the instability region can be determined as the set of all critical plastic strains $\varepsilon_{p i}^{C}$ satisfying the set of non-linear algebraic equations

$$
\dot{\varepsilon}_{p i}^{C}=f_{i}\left(\varepsilon_{p}^{C}\right)=\frac{\Omega\left(\varepsilon_{p}^{C}\right)}{t_{0} Z_{i}^{1 / n}} \quad(i=1,2) .
$$

Taking into consideration that $Z_{1,2}$ are positive and depend on the plastic strain rate, we readily observe that: (i) $\operatorname{sgn}\left(\mathrm{d} f_{1} / \mathrm{d} \varepsilon_{p}^{C}\right)=\operatorname{sgn}\left(\mathrm{d} \Omega / \mathrm{d} \varepsilon_{p}^{C}\right)=\operatorname{sgn}\left(\mathrm{d} f_{2} / \mathrm{d} \varepsilon_{p}^{C}\right)$ for all strains $\varepsilon_{p}^{C}$ and (ii) that there is a set of plastic strain rates with $\varepsilon_{p}^{C}=0$. The first implication shows that for valid instability criterion, it is impossible to model with the same functional dependence, i.e. for a fixed set of material parameters, the inverse behavior for small strain rates and the normal behavior for large strain rates (see Fig. 3). Hence the question of how the strain hardening model can be modified to capture the lower and the upper bounds in the critical strain for the onset of the PLC effect becomes that of crucial importance. Few attempts have been made so far to resolve this issue, unfortunately without a striking success. The forest hardening model (Kubin and Estrin, 1992b) comes closest to achieving this goal. The model predicts the occurrence of the upper and the lower bounds for stability of plastic flow if $\Omega$ is a non-monotonic function of strain. In fact, such non-monotonic behaviour follows from the dislocation evolution model proposed in the cited work. However, experimental evidence on the shape of the function $\Omega\left(\varepsilon_{p}\right)$ is very scarce, and the data collected so far (Ling and McCormick, 1990; Springer and Schwink, 1995) suggest that $\Omega$ increases with strain monotonically. For that case, the forest hardening model predicts the occurrence of 
only a lower critical strain, which shows a normal behavior with the strain rate. In a later paper, Brechet and Estrin (1995) suggested that a transition from normal to inverse behavior can be predicted in a model based on a precipitate-controlled (rather than solute-controlled) deformation mechanism, but this is a rather special case applicable to alloys with shearable precipitates only.

In the present study we follow a different approach and in order to model the both types of behavior we modify the Bilby-Louat term specifying $\sigma_{B}$ without changing the monotonicity of $\Omega$. Assuming that both $\sigma_{B 0}$ and $\Omega$ depend on plastic strain, the instability condition (4) and more precisely the experimentally acquired stability map $\varepsilon_{c}\left(\dot{\varepsilon}_{p}\right)$ can be used to determine both functions. An inspection of the equation

$$
S\left(\varepsilon_{p}, \dot{\varepsilon}_{p}\right)=\dot{\varepsilon}_{p} \frac{\partial \sigma_{F}}{\partial \dot{\varepsilon}_{p}}=0
$$

with $\sigma_{B 0}=\sigma_{B 0}\left(\varepsilon_{p}\right)$ shows that both regimes of dynamic strain aging can be reproduced within this approach. For simplicity we assume in the following that $\sigma_{B 0}$ is an affine transformation of strain. Based on this assumption, we outline in the following sections sections a novel constitutive model, first in the geometrically linear case and then for large strains as well. An interesting question to address would be a comparison of the results obtained using the present model with those reported by Zhang et al. (2001), where it was suggested to introduce a strain rate dependence in the argument of the exponential function of the Cottrell-Bilby-Louat relation. For a monotonically increasing strain dependence of $\Omega$ such a modification results in a faster saturation of the Cottrell-Bilby-Louat term, while by modifying $\sigma_{B 0}$ we only scale the relation thus changing the range of $\sigma_{B 0}$ while keeping the local geometric properties of this contribution unchanged. A strain dependence of the kind introduced by Zhang et al. (2001) affects the predicted critical strain for necking and might results in unreliable forming limit diagrams.

\subsection{Three-dimensional geometrically linear model}

Before formulating the geometrically non-linear model we summarize the basic equations in a geometrically linear setting (small strains). We assume that both the elastic and the viscoplastic response are direction-independent, i.e. isotropic. The stresses are linearized in the elastic strains. The viscoplastic flow is modeled in terms of an overstress model with the flow direction equal to the direction of the stress deviator. The elastic law and the flow rule are given by

$$
\boldsymbol{\sigma}=\lambda \operatorname{tr}\left(\varepsilon_{e}\right) \boldsymbol{I}+2 \mu \varepsilon_{e}, \quad \boldsymbol{\varepsilon}_{e}=\boldsymbol{\varepsilon}-\boldsymbol{\varepsilon}_{p}
$$



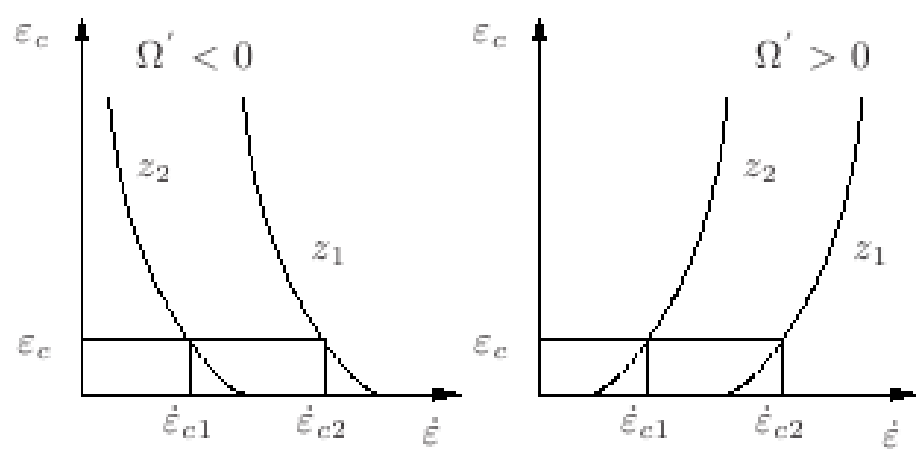

Figure 3: Inverse (left) and normal (right) stability maps

and

$$
\dot{\varepsilon}_{p}=\frac{3}{2} \frac{\dot{\varepsilon}_{p}}{\sigma_{e q}} \boldsymbol{\sigma}^{\prime}, \quad \dot{\varepsilon}_{e q}=\dot{\varepsilon}_{0}\left\langle\frac{\sigma_{e q}-\sigma_{H}\left(\varepsilon_{p}\right)-\sigma_{B}\left(\varepsilon_{p}, t_{a}\right)}{\sigma_{D}}\right\rangle^{m}, \quad\langle x\rangle=\max (0, x),
$$

respectively. $\varepsilon$ is the infinitesimal strain tensor. $\varepsilon_{e}$ and $\varepsilon_{p}$ are the elastic and the plastic parts of $\varepsilon$, respectively. The von Mises equivalent stress is now defined by $\sigma_{e q}=\sqrt{3 / 2}\left\|\boldsymbol{\sigma}^{\prime}\right\|$. The accumulated plastic strain $\varepsilon_{p}=\int_{0}^{t} \sqrt{2 / 3}\left\|\dot{\boldsymbol{\varepsilon}}_{p}\right\| \mathrm{d} \tilde{t}$ determines the stress hardening part of the flow stress through the Voce ansatz

$$
\sigma_{H}\left(\varepsilon_{p}\right)=\sigma_{0}+\left(\sigma_{\infty}-\sigma_{0}\right)\left(1-\exp \left(-\frac{\Theta_{0} \varepsilon_{p}}{\sigma_{\infty}-\sigma_{0}}\right)\right) .
$$

Here, $\sigma_{0}$ and $\sigma_{\infty}$ and denote the initial and the saturation values of the stress and $\theta_{0}$ corresponds to the so called Stage II hardening stage (Estrin and Mecking, 1984).

The dynamic strain aging part is assumed to be given by the Cottrell-Bilby-Louat relation

$$
\sigma_{B}\left(\varepsilon_{p}, t_{a}\right)=\sigma_{B 0}\left(\varepsilon_{p}\right)\left(1-\exp \left(-\left(\frac{t_{a}}{t_{0}}\right)^{n}\right)\right), \quad \sigma_{B 0}\left(\varepsilon_{p}\right)=\sigma_{B 00}+\sigma_{B 00}^{\prime} \varepsilon_{p} .
$$

Here $\sigma_{B 00}$ and $\sigma_{B 00}^{\prime}$ are constants. The evolution of the aging time is (see, e.g., McCormick, 1988; McCormick et al., 1993; Kubin and Estrin, 1990)

$$
\dot{t}_{a}=1-\frac{t_{a}}{t_{w}}, \quad t_{w}=\frac{\dot{\varepsilon}_{p}}{\Omega\left(\varepsilon_{p}\right)}, \quad \Omega\left(\varepsilon_{p}\right)=\Omega_{0}+\Omega_{0}^{\prime} \varepsilon_{p} .
$$

where $\Omega_{0}$ and $\Omega_{0}^{\prime}$ are constants. Note that the aging time $t_{a}$ is no longer set to be identical to $t_{w}$, but rather evolves to the latter as described by a relaxation equation, Eq. (10) (McCormick, 1988).

Where due to the relatively thin range of the map $\Omega\left(\varepsilon_{p}\right)$ (cf. with Ling and McCormick, 1990) an affine approximation of the form $\Omega\left(\varepsilon_{p}\right)=\Omega_{0}+\Omega_{0}^{\prime} \varepsilon_{p}$ is utilized. 


\subsection{Three-dimensional geometrically non-linear model}

In this section we extend the capabilities of the small strain model towards capturing the plastic activity arising in geometrically non-linear setting. We develop an elasticviscoplastic constitutive model capable of describing the normal and inverse behavior of a material under dynamic strain aging conditions. Conceptually, the model is in line with an hyperelastic-viscoplastic type of formulations of material response and it is based upon the assumptions of small elastic strains and finite plastic strains and rotations. In formulating a geometrically non-linear elastic-viscoplastic material model we start with the multiplicative decomposition of the deformation gradient $\boldsymbol{F}$ (see, e.g., Lee, 1969; Mandel, 1974)

$$
\boldsymbol{F}=\boldsymbol{F}_{e} \boldsymbol{F}_{p}
$$

$\boldsymbol{F}_{p}$ is a path-dependent, invertible, non-symmetric tensor. If the material is plastically incompressible then $\operatorname{det}\left(\boldsymbol{F}_{p}\right)=1$ holds. Issues of the non-uniqueness of the decomposition are not addressed here since they are not relevant in the present context. As the elastic strains are assumed to be small, any linear relation between a conjugate pair of generalized stress and strain measures is applicable for the description of the elastic behavior. Here, we assume a linear relation between the 2nd Piola-Kirchhoff stress tensor and the Green strain tensor with respect to the undistorted state. In an Eulerian setting, this ansatz implies that the Kirchhoff stress tensor $\tau$ is given as a linear tensor function of the Almansi strain tensor $\boldsymbol{E}_{e}^{A}$ (see, e.g., Böhlke and Bertram, 2001; Böhlke et al., 2003)

$$
\boldsymbol{\tau}=\mathbb{C}_{e}\left[\boldsymbol{E}_{e}^{A}\right], \quad \boldsymbol{E}_{e}^{A}=\frac{1}{2}\left(\boldsymbol{I}-\boldsymbol{B}_{e}^{-1}\right), \quad \boldsymbol{B}_{e}=\boldsymbol{F}_{e} \boldsymbol{F}_{e}^{\top},
$$

with $\boldsymbol{I}$ being the 2nd-order unit tensor. The Kirchhoff stress tensor $\boldsymbol{\tau}=J \boldsymbol{\sigma}$ is defined in terms of the Cauchy stress tensor $\boldsymbol{\sigma}$ and the determinant $J$ of $\boldsymbol{F}$. The Eulerian stiffness operator $\mathbb{C}_{e}$ is given by the Rayleigh product of $\boldsymbol{F}_{e}$ and the (constant) reference stiffness tensor $\tilde{\mathbb{C}}$ (see, e.g., Bertram, 2005)

$$
\mathbb{C}_{e}=\boldsymbol{F}_{e} \star \tilde{\mathbb{C}}=\tilde{C}_{i j k l}\left(\boldsymbol{F}_{e} \boldsymbol{e}_{i}\right) \otimes\left(\boldsymbol{F}_{e} \boldsymbol{e}_{j}\right) \otimes\left(\boldsymbol{F}_{e} \boldsymbol{e}_{k}\right) \otimes\left(\boldsymbol{F}_{e} \boldsymbol{e}_{l}\right) .
$$

The components $\tilde{C}_{i j k l}$ of $\tilde{\mathbb{C}}$ refer to the fixed orthonormal sample system $\boldsymbol{e}_{i}$. For elastically isotropic materials the reference stiffness $\tilde{\mathbb{C}}$ has the following representation $\tilde{\mathbb{C}}=\lambda \boldsymbol{I} \otimes \boldsymbol{I}+2 \mu \mathbb{I}^{\mathrm{S}}$ with $\lambda$ and $\mu$ denoting the Lamé constants. $\mathbb{I}^{\mathrm{S}}$ is the 4 th-order identity tensor acting on the subspace of all symmetric 2 nd-order tensors. Due to the elastic isotropy, the Eulerian stiffness tensor can be further simplified if the polar decomposition of $\boldsymbol{F}_{e}=\boldsymbol{V}_{e} \boldsymbol{R}_{e}$ is taken into account

$$
\mathbb{C}_{e}=\boldsymbol{F}_{e} \star \tilde{\mathbb{C}}=\left(\boldsymbol{V}_{e} \boldsymbol{R}_{e}\right) \star \tilde{\mathbb{C}}=\boldsymbol{V}_{e} \star\left(\boldsymbol{R}_{e} \star \tilde{\mathbb{C}}\right)=\boldsymbol{V}_{e} \star \tilde{\mathbb{C}}
$$


For the Kirchhoff stress tensor we thus obtain

$$
\boldsymbol{\tau}=\left(\frac{\lambda}{2}\left(\operatorname{tr}\left(\boldsymbol{B}_{e}\right)-3\right) \boldsymbol{I}+\mu\left(\boldsymbol{B}_{e}-\boldsymbol{I}\right)\right) \boldsymbol{B}_{e} .
$$

The last equation shows that $\boldsymbol{\tau}$ and $\boldsymbol{B}_{e}$ are coaxial which is a result of the assumed isotropy of the elastic behavior. If the elastic strains are small then $\boldsymbol{B}_{e} \approx \boldsymbol{I}$ holds and the Kirchhoff stress is given approximately by $\boldsymbol{\tau} \approx \lambda\left(\operatorname{tr}\left(\boldsymbol{B}_{e}\right)-3\right) \boldsymbol{I} / 2+\mu\left(\boldsymbol{B}_{e}-\boldsymbol{I}\right)$, which implies for the stress deviator $\boldsymbol{\tau}^{\prime} \approx \mu \boldsymbol{B}_{e}^{\prime}$.

The Kirchhoff stress tensor is completely determined by the symmetric variable $\boldsymbol{B}_{e}$ for which an symmetry preserving evolution equation has to be formulated. The material time derivative of $\boldsymbol{B}_{e}$ is

$$
\dot{\boldsymbol{B}}_{e}=\dot{\boldsymbol{F}}_{e} \boldsymbol{F}_{e}^{\top}+\boldsymbol{F}_{e} \dot{\boldsymbol{F}}_{e}^{\top}
$$

Similar to crystal plasticity, the rate of change of $\boldsymbol{F}_{p}$ is assumed to depend on the Mandel stress tensor $\boldsymbol{T}_{e}=\boldsymbol{C}_{e} \boldsymbol{S}_{e}$ and a set of internal variables $\boldsymbol{\alpha}=\left\{\varepsilon_{p}, t_{a}\right\}$

$$
\dot{\boldsymbol{F}}_{p} \boldsymbol{F}_{p}^{-1}=\tilde{\boldsymbol{k}}\left(\boldsymbol{T}_{e}^{\prime}, \boldsymbol{\alpha}\right)
$$

$\boldsymbol{S}_{e}$ is the 2nd Piola-Kirchhoff stress tensor and $\boldsymbol{C}_{e}=\boldsymbol{F}_{e}^{\top} \boldsymbol{F}_{e}$ the right Cauchy-Green tensor. In the following we assume that the plastic behavior is isotropic. Hence, $\tilde{\boldsymbol{k}}$ is an isotropic tensor function. Combining Eqs. (16) and (17) and making use of the elastic and the plastic isotropy we find

$$
\mathcal{L}\left(\boldsymbol{B}_{e}\right)=\dot{\boldsymbol{B}}_{e}-\boldsymbol{L} \boldsymbol{B}_{e}-\boldsymbol{B}_{e} \boldsymbol{L}^{\top}=-2 \operatorname{sym}\left(\tilde{\boldsymbol{k}}\left(\boldsymbol{\tau}^{\prime}, \boldsymbol{\alpha}\right)\right) \boldsymbol{B}_{e}
$$

with the spatial velocity gradient $\boldsymbol{L}=\partial \boldsymbol{v} / \partial \boldsymbol{x}$. By analogy to the geometrically linear model, we assume for the flow rule

$$
\tilde{\boldsymbol{k}}\left(\boldsymbol{\tau}^{\prime}, \boldsymbol{\alpha}\right)=\frac{3}{2} \frac{\dot{\varepsilon}_{p}}{\sigma_{e q}} \boldsymbol{\tau}^{\prime}
$$

The equivalent von Mises stress and the equivalent plastic strain rate are defined by $\sigma_{e q}=\sqrt{3 / 2}\left\|\boldsymbol{\tau}^{\prime}\right\|$ and $\dot{\varepsilon}_{p}=\sqrt{2 / 3}\|\tilde{\boldsymbol{k}}\|$, respectively. The plastic strain rate is related to the variables $\sigma_{e q}, \sigma_{H}\left(\varepsilon_{p}\right)$ and $\sigma_{B}\left(\varepsilon_{p}, t_{a}\right)$ in the same way as in the geometrically linear model.

\section{Numerical implementation}

\subsection{Numerical time integration scheme}

In order to obtain an incrementally objective time integration scheme the evolution equation for $\boldsymbol{B}_{e}$ is transformed back to the reference configuration of the body. Using 
$\boldsymbol{B}_{e}=\boldsymbol{F} \boldsymbol{G}_{p} \boldsymbol{F}^{\top}$ and $\mathcal{L}_{v} \boldsymbol{B}_{e}=\boldsymbol{F} \dot{\boldsymbol{G}}_{p} \boldsymbol{F}^{\top}$ one further obtains

$$
\dot{\boldsymbol{G}}_{p} \boldsymbol{G}_{p}^{-1}=-2 \boldsymbol{F}^{-1} \operatorname{sym}\left(\tilde{\boldsymbol{k}}\left(\boldsymbol{\tau}^{\prime}, \boldsymbol{\alpha}\right)\right) \boldsymbol{F} .
$$

The variable $\boldsymbol{G}_{p}=\boldsymbol{F}_{p}^{-1} \boldsymbol{F}_{p}^{-\top}$ satisfies $\operatorname{det}\left(\boldsymbol{G}_{p}\right)=1$ in the plastically incompressible case. Equation (20) implies that in this case the evolution equation (17) has to obey the constraint $\operatorname{tr}(\tilde{\boldsymbol{k}})=0$, which is satisfied by the flow rule given by Eq. (19). In the following, all variables at time $t_{n}$ have an index $n$. Variables without index refer to time $t_{n+1}$ with $\Delta t=t_{n+1}-t_{n}$. The (implicit) exponential map (Simo and Miehe, 1992),

$$
\boldsymbol{G}_{p}=\exp \left(\dot{\boldsymbol{G}}_{p} \boldsymbol{G}_{p}^{-1} \Delta t\right) \boldsymbol{G}_{p}^{n}
$$

fulfils the incompressibility constraint for $\boldsymbol{G}_{p}$ exactly. Because of the invariance of $\boldsymbol{G}_{p}$ under superimposed rigid body motions, the time integration rule is incrementally objective. Transforming Eq. (21) back to the current configuration of the body and taking into account the properties of the tensorial exponential function, one obtains for the flow rule

$$
\boldsymbol{B}_{e}=\exp \left(-2 \operatorname{sym}\left(\tilde{\boldsymbol{k}}\left(\boldsymbol{\tau}^{\prime}, \boldsymbol{\alpha}\right)\right) \Delta t\right) \hat{\boldsymbol{B}}_{e}=\exp \left(-2 \Delta \gamma \boldsymbol{N}^{\prime}\right) \hat{\boldsymbol{B}}_{e}
$$

with the trial variable $\hat{\boldsymbol{B}}_{e}=\boldsymbol{F} \boldsymbol{G}_{p}^{n} \boldsymbol{F}^{\top}$, the stress direction $\boldsymbol{N}^{\prime}=\boldsymbol{\tau}^{\prime} /\left\|\boldsymbol{\tau}^{\prime}\right\|$ and $\Delta \gamma=\sqrt{3 / 2} \Delta t \dot{\varepsilon}_{p}=\sqrt{3 / 2} \Delta \varepsilon_{p}$. Since $\boldsymbol{\tau}^{\prime}$ is coaxial with $\boldsymbol{B}_{e}$ in the isotropic elastic case, $\boldsymbol{B}_{e}$ and $\hat{\boldsymbol{B}}_{e}$ are coaxial too. In contrast to the geometrically linear case, no proportionality between the unknown and the trial variables is obtained. In the following we simplify the update formula drastically by consequently exploiting the smallness of the elastic strains. For small $\Delta \gamma$ and small elastic strains one obtains

$$
\hat{\boldsymbol{B}}_{e}=\exp \left(2 \Delta \gamma \boldsymbol{N}^{\prime}\right) \boldsymbol{B}_{e} \approx\left(\boldsymbol{I}+2 \Delta \gamma \boldsymbol{N}^{\prime}\right) \boldsymbol{B}_{e} \approx \boldsymbol{B}_{e}+2 \Delta \gamma \boldsymbol{N}^{\prime}
$$

and similarly

$$
\hat{\boldsymbol{B}}_{e}^{\prime}=\boldsymbol{B}_{e}^{\prime}+2 \Delta \gamma \boldsymbol{N}^{\prime}
$$

This equation does no longer satisfy $\operatorname{det}\left(\boldsymbol{B}_{e}\right)=\operatorname{det}(\boldsymbol{B})$ or equivalently $\operatorname{det}\left(\boldsymbol{F}_{p}\right)=1$ since the exponential function has been approximated. When evaluating this result it has to be taken into account that in the context of small elastic strains and hence small total volume changes the approximation $\operatorname{det}(\boldsymbol{B}) \approx 1+(\operatorname{tr}(\boldsymbol{B})-3)$ holds. Hence, $\operatorname{tr}(\boldsymbol{B}) \approx \operatorname{tr}\left(\boldsymbol{B}_{e}\right)$ is valid. With the approximate form of the elastic law $\boldsymbol{\tau}^{\prime} \approx \mu \boldsymbol{B}_{e}^{\prime}$ and the trial stress $\hat{\boldsymbol{\tau}}^{\prime}=\mu \hat{\boldsymbol{B}}_{e}^{\prime}$ equation (23) becomes $\hat{\boldsymbol{\tau}}^{\prime}=\boldsymbol{\tau}^{\prime}+2 \mu \Delta \gamma \boldsymbol{N}^{\prime}$. Now similarly to the small strain case, $\tau^{\prime}$ is proportional to $\hat{\tau}^{\prime}$ and one arrives at

$$
\left\|\hat{\boldsymbol{\tau}}^{\prime}\right\|=\left\|\boldsymbol{\tau}^{\prime}\right\|+2 \mu \Delta \gamma
$$


with $\|\boldsymbol{A}\|=\sqrt{\operatorname{tr}\left(\boldsymbol{A} \boldsymbol{A}^{\top}\right)}$. From Eq. (25) it follows

$$
\hat{\sigma}_{e q}=\sigma_{e q}+3 \mu \Delta \varepsilon_{p}
$$

In the overstress model, $\sigma_{e q}$ can be eliminated

$$
\hat{\sigma}_{e q}=\sigma_{D}\left(\frac{1}{\dot{\varepsilon}_{0}} \frac{\Delta \varepsilon_{p}}{\Delta t}\right)^{\frac{1}{m}}+\sigma_{H}\left(\varepsilon_{p}^{n}+\Delta \varepsilon_{p}\right)+\sigma_{B}\left(\varepsilon_{p}^{n}+\Delta \varepsilon_{p}, t_{a}^{n}+\Delta t_{a}\right) .
$$

The aging time is integrated by the Euler backward scheme

$$
t_{a}=t_{a}^{n}+\Delta t \dot{t}_{a}=t_{a}^{n}+\Delta t-\frac{t_{a} \Delta \varepsilon_{p}}{\Omega\left(\varepsilon_{p}^{n}+\Delta \varepsilon_{p}\right)}
$$

Although an implicit integration scheme is applied, the aging time $t_{a}$ can be explicitly given as a function of $\Delta \varepsilon_{p}$

$$
t_{a}=\frac{t_{a}^{n}+\Delta t}{1+\Delta \varepsilon_{p} / \Omega\left(\varepsilon_{p}^{n}+\Delta \varepsilon_{p}\right)} .
$$

It can be seen that the implicit Euler scheme ensures a non-negative aging time. Since the aging time $t_{a}$ at $t_{n+1}$ is determined explicitly, it can be eliminated from equation (27). As a result, at a time step only one nonlinear algebraic equation needs to be solved. The constitutive equations were implemented through an implicit integration procedure in ABAQUS/Standard utilizing the interface and programming capabilities provided for user defined material models (ABAQUS/Standard, 2003, UMAT subroutines). In the geometrically linear case the consistent linearization of the incremental form of the constitutive equations delivers an algorithmic tangent with a modified structure that resembles the standard algorithmic tangent of the classical isotropic von Mises plasticity. In the geometrically non-linear case the modified tangent was computed on the basis of numerical linearization of the constitutive model again employing the user material subroutines in ABAQUS.

\subsection{Parameter identification}

Material parameters necessary for calibration of the proposed constitutive models have been identified on the basis of a comparison between the simulation and experiment. For this purpose we simulated in several steps the tensile experiments discussed above (see Section 2) and compared the relevant segments of the resulting response diagrams. The material parameters of the constitutive model were identified so as to reproduce the experimentally observed intermediate stress distribution computationally. In a second step the material parameters in the Cottrell-Bilby-Louat contribution were estimated iteratively, along with the strain hardening parameter $\theta_{0}$, so that the computed normal and the inverse behavior of the $\mathrm{Al}$ alloy fit the experimentally observed one. 
The parameters responsible for the normal and inverse behavior could then be estimated on the basis of the simple, one-dimensional, rigid-viscoplastic constitutive model presented. It was possible to determine the instability range from the rigidviscoplastic model using the condition $\dot{\varepsilon}_{p} \partial \sigma_{F} / \partial \dot{\varepsilon}_{p}=0$. Using the estimated strain hardening parameter $\theta_{0}$ the stress amplitude $\sigma_{B 0}$ and the characteristic time $t_{0}$ were then calculated for every strain level using the Newton-Raphson method.

The values predicted by the constitutive model are shown in Fig. 4 and Fig. 5. The agreement between the experimental and the computed stability maps on Fig. 5 is remarkable.

\begin{tabular}{c|c|c|c|c|c}
\hline \hline$E$ & $70.0 \mathrm{GPa}$ & $\dot{\varepsilon}_{0}$ & $3.5 \cdot 10^{-5} 1 / \mathrm{s}$ & $\sigma_{B 00}$ & $18.9 \mathrm{MPa}$ \\
$\nu$ & 0.3 & $\sigma_{D}$ & $15.0 \mathrm{MPa}$ & $\sigma_{B 00}^{\prime}$ & $189.26 \mathrm{MPa}$ \\
& & $m$ & 28.0 & $\Omega_{0}$ & $6.81 \cdot 10^{-4}$ \\
& & $\sigma_{0}$ & $123.0 \mathrm{MPa}$ & $\Omega_{0}^{\prime}$ & $3.6 \cdot 10^{-4}$ \\
& & $\sigma_{\infty}$ & $343.0 \mathrm{MPa}$ & $t_{0}$ & $0.125 \mathrm{~s}$ \\
& & $\Theta_{0}$ & $2800.0 \mathrm{MPa}$ & $n$ & $1 / 3$ \\
\hline \hline
\end{tabular}

Table 1: Material parameters

\section{Results and discussion}

\subsection{Statistical analysis}

The PLC effect in polycrystalline materials, such as Al alloy 2024 considered here, is typically accompanied with complex deformation patterns discontinuous in time and localized in space. As previously mentioned, different types of spatiotemporal strain localization bands (stress serrations in the response diagram) can be observed under the PLC conditions. The bands of type $C$ can be considered 'temporally static' but 'weakly spatially correlated'. They occur at low strain rates at weakly correlated positions along a flat sample. Type B instabilities were also static and occurred at intermediate strain rates, the stress drops being distributed more densely along the time axis. In addition, we found that new localized bands were formed near the old ones. The latter fact is an indication of the existence of a spatial correlation between the band nucleation events. At high strain rates the type B instabilities transformed to type A PLC bands with stress drops occurring in a faster time sequence. The bands propagated along the tensile axis of the sample in an apparently continuous manner, which is an indication of nucleation events being strongly correlated in space. Recent studies showed that this complex spatiotemporal behavior emerging as a result of an unsta- 

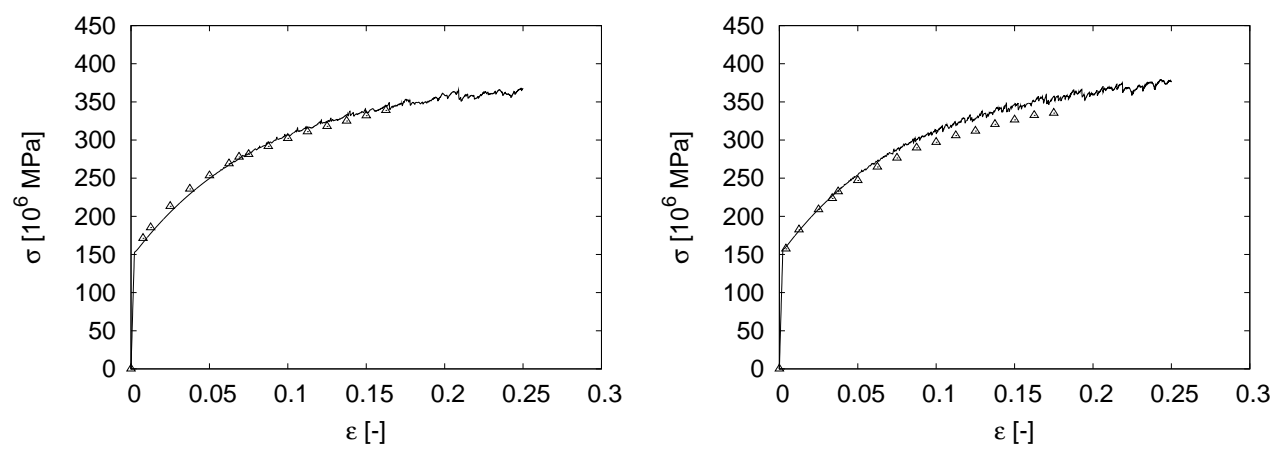

a) $\dot{\varepsilon}_{a}=1 \cdot 10^{-1} s^{-1}$

b) $\dot{\varepsilon}_{a}=1 \cdot 10^{-2} s^{-1}$
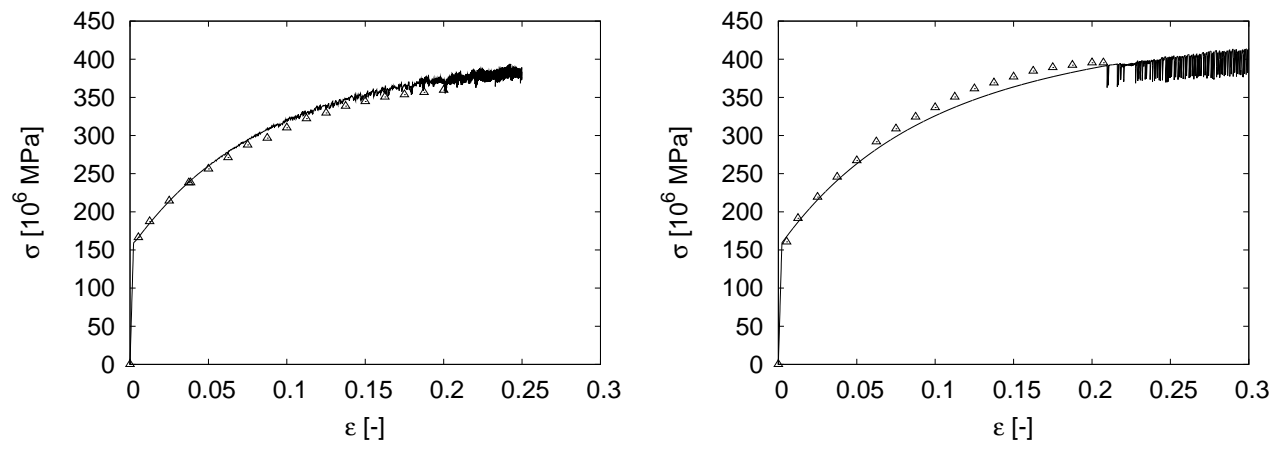

c) $\dot{\varepsilon}_{a}=1 \cdot 10^{-3} s^{-1}$

d) $\dot{\varepsilon}_{a}=1 \cdot 10^{-4} s^{-1}$

Figure 4: Experimental (global) and simulation results: true strain vs true stress
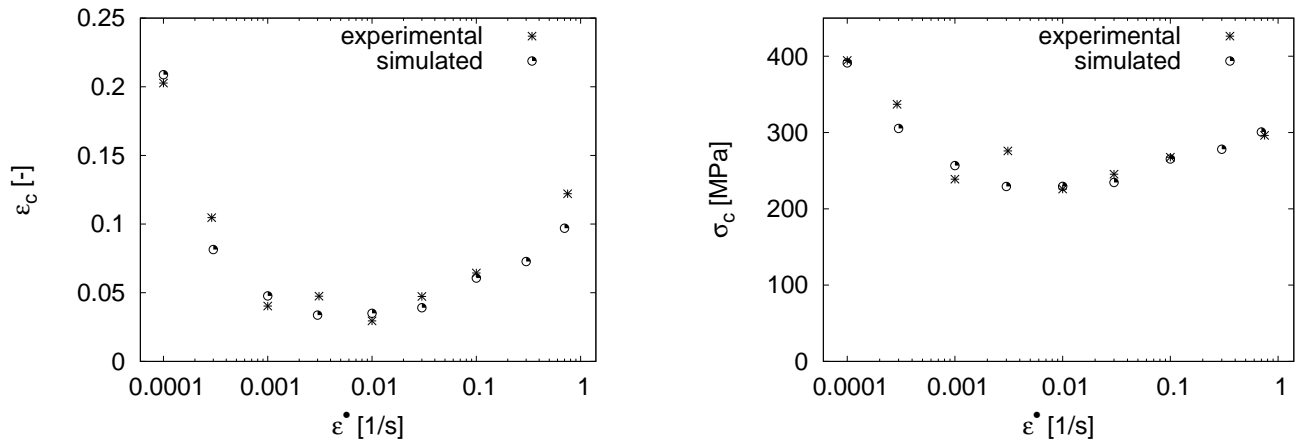

Figure 5: Experimental and simulation results: stability maps

ble plastic flow, and especially the transition from uncorrelated to strongly correlated band nucleation events, is a consequence of the spatiotemporal interaction between self-organized (dissipative) dislocation structures. It was also proven (Bharathi et al., 2001) that the transition from a dynamic chaos (bands of type B) to a self-organized critical behavior (bands of type A) indeed happens in materials exhibiting the PLC effect, and that moreover the transition between these two distinct dynamical regimes is con- 
trolled by a single scalar parameter: the effective applied strain rate. In the following, we report the results from an investigation of the computationally simulated statistics of the 'catastrophic slip' associated with the PLC effect and calculate the two-point temporal correlation function for the attendant serrations on the stress-strain curve. The aim of this exercise is:

a) to test the capabilities of the constitutive model developed in order to predict the transition from chaos to self organized criticality based on calculated two-point correlation function;

b) to show that our material model is in agreement with the available results of a statistical analysis of the experimentally observed discontinuous yielding, thus predicting a change in the peaked distribution of the magnitude of stress drops toward a monotonic one with the increase of the applied strain rate.

In order to achieve these aims, we first calculated an 'averaged' stress-strain curve. The difference between an averaged stress-strain curve and the experimentally determined one gives a statistically representative sample of strain-hardening independent stress fluctuations. After suppressing the ascending fluctuations and retaining the stress drops one can see readily that the simulated stress-time series, resemble the experimentally recorded stress-time series (see e.g. Fig. 4). Typical fragments of a stress-strain curve obtained for four different strain rates are shown in Fig. 6 a) - c). We have analyzed the distribution of the normalized stress drop magnitudes for every individual segment of the diagram. The normalization was performed on the basis of the standard linear regression fit $\overline{\Delta \sigma}=f(\varepsilon)$ for the statistics of the stress drops $\triangle \sigma$ versus strain $\varepsilon$, and the resulting distribution of the normalized stress drops $\delta=\triangle \sigma / \overline{\triangle \sigma}$ was considered. The resulting histograms - the number of stress drops $Z$ versus normalized stress drop magnitude $\delta$ - are shown in Fig. 6. Since the strain rate increases from the bottom to the top of Fig. 6, a trend in the histograms and hence in the distribution functions for the normalized stress drop magnitudes is recognized easily: they tend to transform from a bi-modal form (bands of type C) to a power law distribution $Z \sim \delta^{-p}$ (bands of type A). As no attempt was made here to estimate $p$, we refer the interested reader to a more detailed statistical analysis (Lebyodkin et al., 2001). To summarize the results so far, we conclude that the proposed material model does predict a change of a peaked distribution of stress drops to a monotonic one and hence proves to be in a qualitative agreement with the available experimental data and the aforementioned statistical studies. 
The two-point correlation function (see Fig. 7 a) - c)) gives an insight into the temporal distribution of the band nucleation events in the simulated time interval of the PLC effect, of course under controlled strain rate. More precisely, the questions we are interested in addressing are: (i) given a band nucleation event at the temporal instant $t_{1}$ in the simulated time interval, what is the probability of there being another nucleation event at a second time point $t_{2}$ in the same interval? and (ii) how does the length of the simulated time interval affect the distribution of the nucleation events? The answers to both questions are expressed graphically in Fig. 7 a) - c). The diagrams of the calculated two-point temporal correlation function show that the higher the strain rate, the longer is the time interval $\tau$ in which a pair of nucleation events can be considered as being correlated. In a very simple and illustrative way these results indicate the plausibility of the conclusion drawn by Bharathi et al. (2001) that the increase in the strain rate leads to a change in the dynamical regime of the plastic flow from a non-linear chaotic (nucleation events with short range of correlation) to a self-organized critical behavior (nucleation events with long-range correlation).

\subsection{Band characteristics in tension and compression}

In this section we determine the most important characteristics of the PLC bands of type A, viz. the band velocity and the band width, from the calculated plastic response. A typical band of type A occurs as a plastic strain-rate spike propagating along the tensile axis of the specimen as a solitary plastic wave. The projected uniaxial motion of the solitary plastic wave is realized through a repetitive micromechanical mechanism consisting of four phases: in the first phase the dislocations are unpinned from the solute clouds; the second phase is characterized by a rapid dislocations glide; in the third phase the dislocations are recaptured and pinned at the forest obstacles; and finally in the fourth phase additional pinning by solutes occurs. Changes in the strain field within the band are associated with the second phase while the strain rate is affected by the micromechanics of the third phase of band propagation and exhibits sharp variations across the band width (Rizzi and Hähner, 2004). This temporal picture translates to spatial variation in the plastic strain rate (and hence in the plastic strain) within the band. The simulation results shown on Fig. 8 support this conjecture. In order to estimate the band characteristics we assume, as is done customarily (Hähner et al., 2002), that

i) the band plastic strain accommodates the total applied strain; 

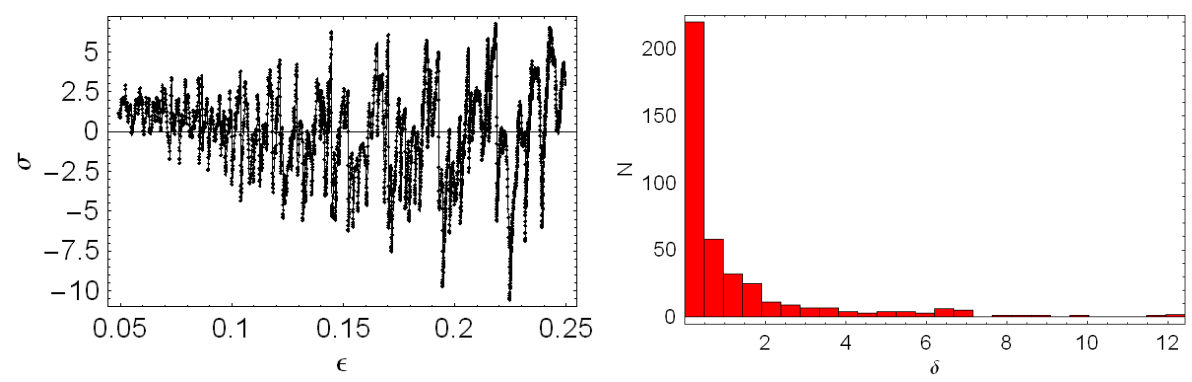

a) $\dot{\varepsilon}_{a}=1 \cdot 10^{-2} s^{-1}$
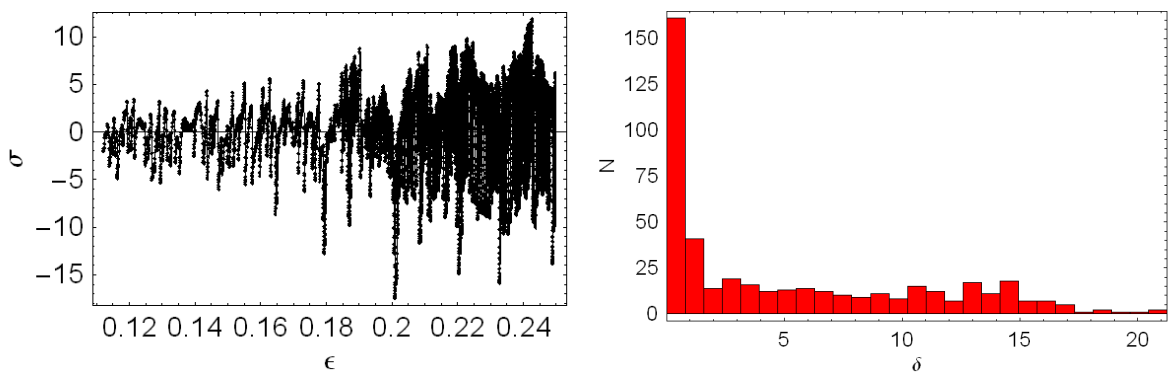

b) $\dot{\varepsilon}_{a}=1 \cdot 10^{-3} s^{-1}$
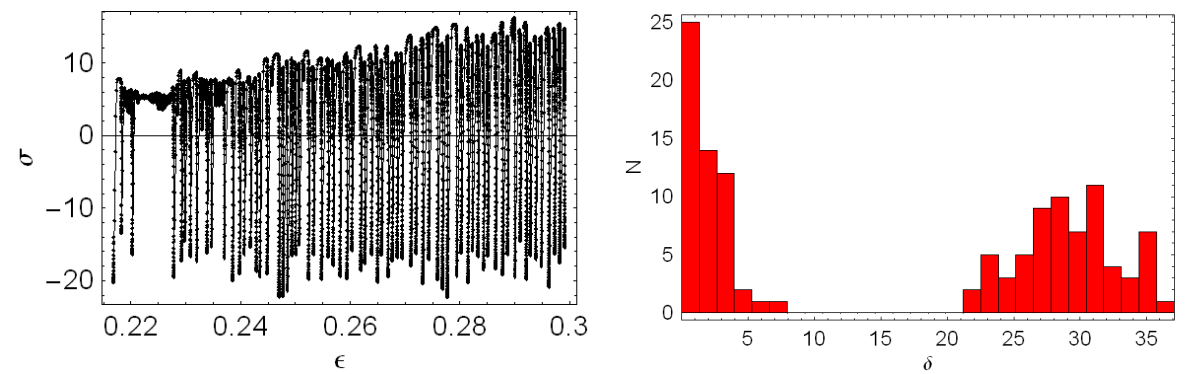

c) $\dot{\varepsilon}_{a}=1 \cdot 10^{-4} s^{-1}$

Figure 6: Simulated $\sigma-\varepsilon$ diagrams and histograms of the normalized stress drop magnitude $\delta$. Here $Z$ denotes the number of the stress drops at with magnitude $\delta$.

ii) the effect of the stress rate on the motion of an already developed PLC band is negligible.

To determine both spatial characteristics of the localized deformation bands mentioned above, we conducted numerical simulations of uniaxial tension/compression experiments. The plastic activity is characterized in terms of the equivalent plastic strain increment $\triangle \varepsilon_{p}$. Figure 8 shows the distribution of this increment along the tensile/compression axis $x$ of the specimen. The values of the increment are obtained at different time instants (increments) during the band propagation. From the axial displacement at the peaks of the equivalent plastic strain increment and the corresponding time intervals one can determine the velocity of band propagation. Indeed, it should 


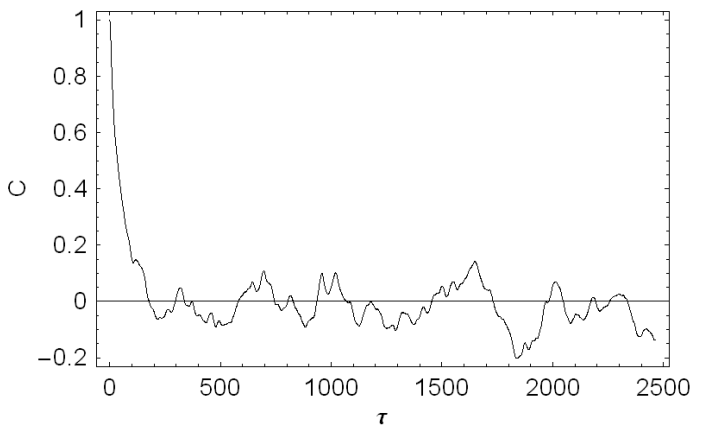

a) $\dot{\varepsilon}_{a}=1 \cdot 10^{-2} s^{-1}$

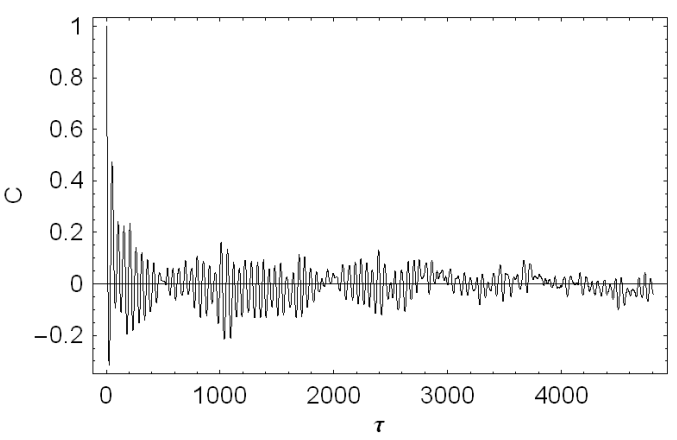

b) $\dot{\varepsilon}_{a}=1 \cdot 10^{-3} \mathrm{~s}^{-1}$

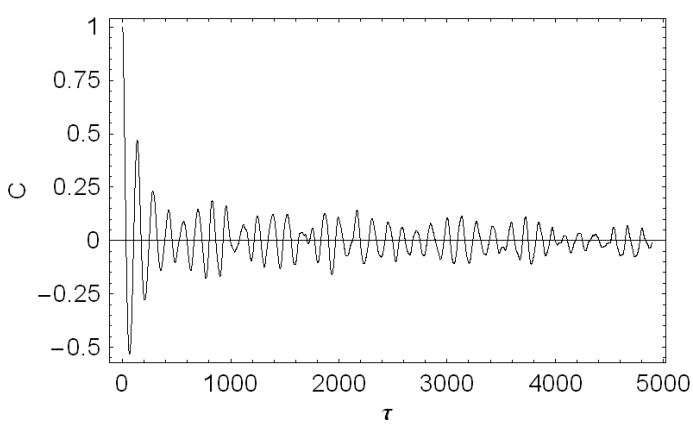

c) $\dot{\varepsilon}_{a}=1 \cdot 10^{-4} s^{-1}$

Figure 7: Two-point correlation functions.

be noted that assumption (a) implies for the band velocity ${ }^{2} c$ that $c=\triangle \varepsilon_{p}^{*} / v$ where $v$ is the cross-head velocity, calculated as $v=\left.u\right|_{\triangle \varepsilon_{p}^{*}} / \triangle t$, with $\left.u\right|_{\triangle \varepsilon_{p}^{*}}$ denoting the component of the displacement field in direction $x$ corresponding to the maximum value $\triangle \varepsilon_{p}^{*}$ of $\triangle \varepsilon_{p}$, and $\triangle t$ is the time increment for $\triangle \varepsilon_{p}$. In order to determine the (average) band width $w_{x}$ in $x$ direction we again consider the simplifying assumption (a) from which it follows $w_{x}=v / \varepsilon_{p, t}$ with $\varepsilon_{p, t}=\mathrm{d} \varepsilon_{p} / \mathrm{d} t$. Clearly, then $w_{x} \approx v \Delta t / \Delta \varepsilon_{p}^{*}$. holds. The band width in the direction normal to the middle band plane ${ }^{3}$ is calculated using the angle

\footnotetext{
${ }^{2}$ The band velocity is identified with the $x$-coordinate of the total band velocity field.

${ }^{3}$ Assuming the middle band surface to be flat.
} 
$\alpha$ between the unit direction vector $\boldsymbol{e}_{x}$ of the $x$ axis and the unit normal to to the mean band plane (determined from the calculations). Accordingly, the band width in the direction perpendicular to the middle band plane is $w \approx w_{x} \cos (\alpha)$. Figure 8 illustrates the dependence of the band velocity $c$ and the band width $w$ on the applied strain rate $\dot{\varepsilon}_{a}$ (proportional to cross-head velocity $v$ ) graphically. The calculated band width and the band velocity lie in the experimentally observed ranges (Zhang et al., 2001). The graphics in Fig. 8 b) - c) show a linear dependence (note the logarithmic scale!) of band velocity on the applied strain rate and a relative independence of $c$ on the specifics of the test (tension or compression). By contrast, the band width depends strongly on the type of the test and changes only slightly with the cross-head velocity.

Figure 9 a), b) shows the plastic strain increment, the internal variables as well as the von Mises equivalent stress as obtained by simulations of tension/compression experiments with flat samples. For the tensile tests considered the band moves from bottom to top, while for the compression tests the motion is in the opposite direction. The simulations show that in tensile experiments single bands or $\mathrm{X}$-shaped band pairs emerge at a band angle typical of the tensile test. This is distinct from the compression tests where V-shaped band pairs appear. The domains occupied by the bands are easily recognized as the regions of the localized plastic strain. The aging time in the region of a band takes the smallest values $\left(t_{a}=0\right)$. The distribution of the von Mises stress shows that the equivalent stress close to the band front is larger than the equivalent stresses behind the band.

In addition to the tensile tests with flat specimens, results for cylindrical specimens undergoing tension and compression were also obtained (see Fig. 10). The axial symmetry was used in the simulations. In these simulations, small regularly distributed initial fluctuations in the plastic strain field with controlled effective value were introduced at the integration points of the finite element mesh. In this way the uniformity of the plastic flow was perturbed and localized PLC bands were activated as a consequence. The predicted apparent band shape for both tension and compression was conical (Fig. 10). These results are consistent with the available computational data for cylindrical samples (Zhang et al., 2001) and with the experimental observations (McCormick et al., 1993; Ling and McCormick, 1993; van den Brink et al., 1977).

\subsection{Rôle of the dimensionality of the strain state}

In order to show the influence that the dimensionality of the strain state has on the band characteristics, we performed a deformation driven simulation of the isothermal response of a 2-dimensional representative volume element (RVE) (see Fig. 12). The 
RVE was loaded by periodic boundary conditions (displacements) generated from a prescribed averaged strain, for a mesh of 50x50 finite elements. This particular choice of loading conditions was motivated by the observation that periodic boundary conditions result in a softer response of the deformed finite element mesh yielding in result to a localized deformation modes. The strain hardening function applied in the computations is defined in Section 3.2. Different boundary conditions have been generated for principal strain states with varying axiality. The control over the axiality was enforced through the scalar parameter $\alpha$ and the relation $\varepsilon_{2}=\alpha \varepsilon_{1}$ (cf. with Gänser et al., 2000). In the latter, $\alpha=-0.5$ corresponds roughly to a pure tension, $\alpha=0$ to plane strain state and $\alpha=1$ to a biaxial tension. For every $\alpha$ in the discrete set $\alpha \in[-0.5,-0.375,-0.25,-0.125,0,0.25,0.5,1]$ we monitored the deformation up to the appearance of PLC-bands. The obtained results for band orientation angle, in dependence on the axiality parameter $\alpha$ are presented in Table 2 and in Figure 11.

\begin{tabular}{|c|c|c|c|c|c|c|c|c|}
\hline$\alpha$ & -0.5 & -0.375 & -0.25 & -0.125 & 0.0 & 0.25 & 0.5 & 1.0 \\
Orientation angle & $53.0^{\circ}$ & $58.5^{\circ}$ & $62.0^{\circ}$ & $71.0^{\circ}$ & $90.0^{\circ}$ & $90.0^{\circ}$ & - & - \\
\hline
\end{tabular}

Table 2: Simulated dependence of the band orientation angle (in, $\left.\left[{ }^{\circ}\right]\right)$, from the axiality of the strain state (controlled by the parameter $\alpha$ ).

\subsection{Deep drawing of a metal sheet}

An example of an industrial application we analyzed is that of deep drawing of an initially circular sheet of Al alloy 2024, for which the isotropic material properties are assumed. It should be noted that the assumed plastic isotropy precludes the occurrence of earing of a deep drawn sample, although this effect was observed experimentally. Hence, the simulation results shown have a qualitative character only. With the defined constitutive model, the localization pattern observed from the simulation is shown in Fig. 13. The simulation predicts propagating localization bands in circumferential direction along the region with highest mean curvature. The explanation of the observed phenomenon is related to decreased load bearing capacity of the deep drawn sample. As well known from the theory of shells, an extreme loading capacity can be achieved when the geometric design of the shell allows it to be in a membrane state with equally strained material fibers. Clearly in a deep drawing process where the thickness of the metal sheet decreases such a state cannot be achieved. Indeed, the deep drawn sample is deformed in a bending dominated regime that is accompanied by large mid surface inextensional deformations favoring early activation and, more importantly, a non-uniform destabilized character of plastic flow. 


\section{Summary and Conclusions}

The experimental and computational results of the present investigation predicted the onset and the propagation of localized Portevin-Le Chatelier bands in Al alloy 2024 in a qualitatively and quantitatively correct way. The conditions for the occurrence of the inverse and the normal strain rate behavior of the critical strain for the onset of discontinuous plastic flow were determined and calculated using non-linear finite element analysis. The predicted critical strain for the onset of the PLC effect is in good quantitative agreement with the available experimental results. Both the normal and the inverse types of behavior were predicted. For constant strain rate tests, normal behavior is associated with PLC bands of type A, while inverse behavior is associated with type $\mathrm{C}$ bands. Complementary statistical analysis was carried out on the computed and experimentally recorded stress-time series under the dynamic strain aging conditions with the purpose of characterizing the distinct spatiotemporal dynamical regimes associated with the PLC effect. The two-point temporal correlation function was calculated for several different applied strain rates. The results indicate clearly that the present physically and geometrically non-linear constitutive model is able to capture the transition between the distinctly different dynamic regimes of the PLC flow - from non-linear chaos to self-organized criticality. As predicted by the model, the character of the band nucleation events changes from weakly to strongly correlated one as the band type structure of the plastic flow switches from type $\mathrm{C}$ at small strain rates to type A for high strain rates. While the model was gauged to a particular Al alloy (AA 2024), it possesses generality that makes it applicable to a broad range of dynamically strain aging materials.

\section{References}

ABAQUS/Standard, 2003. Hibbitt, Karlsson \& Sorensen, Inc.

Bell, J., 1973. The Experimental Foundations of Solid Mechanics. Vol. VIa/1 of Encyclopedia of Physics. Springer.

Bertram, A., 2005. Elasticity and Plasticity of Large Deformations. Springer-Verlag, Berlin Heidelberg.

Bharathi, M., Lebyodkin, M., Ananthakrishna, G., Fressengeas, C., Kubin, L., 2001. Multifractal Burst in the Spatiotemporal Dynamics of Jerky Flow. Physical Review Letters 87 (16), 165508-1-165508-4. 
Böhlke, T., Bertram, A., 2001. The evolution of Hooke's law due to texture development in polycrystals. Int. J. Solids Struct. 38 (52), 9437-9459.

Böhlke, T., Bertram, A., Krempl, E., 2003. Modeling of deformation induced anisotropy in free-end torsion. Int. J. Plast. 19, 1867-1884.

Brechet, Y., Estrin, Y., 1995. On the Influence of Precipitation on the Portevin-Le Chatelier Effect. Acta metall. mater. 43 (3), 955-963.

Cottrell, A., 1953a. Dislocations and plastic flow in crystals. University Press, Oxford.

Cottrell, A., 1953b. A note on the portevin-le chatelier effect. Philosophical Magazine 44, 829-832.

Cottrell, A., Bilby, B., 1949. Dislocation theory of yielding and strain ageing of iron. Proc. Phys. Soc. London Sect. A 62, 49-62.

Dablij, M., Zeghloul, A., 1997. Portevin-Le Chatelier plastic instabilities: characteristics of deformation bands. Materials Science and Engineering A 237, 1-5.

Dierke, H., Krawehl, F., Graff, S., Forest, S., Ŝachl, J., Neuhäuser, H., 2007. Portevin-Le Chatelier effect in Al-Mg alloys: Influence of obstacles - experiments and modelling. Computational Materials Science 39 106-112.

Estrin, J., 1987. Stoffgesetze der plastischen Deformation und Instabilit "aten des plastischen Fließens. In: VDI Forschungsheft. No. 642. VDI Verlag GmbH, pp. 1-48.

Estrin, Y., Kubin, L., 1995. Spatial Coupling and Propagative Plastic Instabilities. Ed. Mühlhaus, H.-B., New York: Willey.

Estrin, Y., Mecking, H., 1984. A unified phenomenological description of work hardening and creep based on one parameter models. Acta Metall. , 57-70.

Gänser, H.-P., Werner, E., Fischer, F., 2000. Forming Limit diagrams: a micromechanical approach. International Journal of Mechanical Sciences 42, 2042-2054.

Hähner, P., 1993. Modelling the spatio-temporal aspects of the Portevin-Le Châtelier effect. Materials Science and Engineering A 164, 23-34.

Hähner, P., Ziegenbein, A., Rizzi, A., Neuhäuser, H., 2002. Spatiotemporal analysis of Portevin-Le Chatelier deformation bands: Theory, simulation and experiment. Physical Rewiew B 65134109.

Head, A., Louat, N., 1955. The Distribution of Dislocation in Linear Arrays. Australian Journal of Physics 8, 1-7. 
Kubin, L., Chihab, K., Estrin, Y., 1988. The rate dependence of the Portevin-Le Chatelier effect. Acta Metallurgica 36 (10), 2707-2718.

Kubin, L., Estrin, Y., 1990. Evolution of Dislocation Densities and the Critical Conditions for the Portevin-Le Chatelier Effect. Acta metall. mater. 38 (5), 697-708.

Kubin, L., Estrin, Y., 1992a. The Critical Conditions of Jerky Flow. phys. stat. sol (b) 172,173, 173-185.

Kubin, L., Estrin, Y., 1992b. The crytical conditionis for jerky flow. Physica Status Solidi (b) $172,173-185$.

Lebyodkin, M., Brechet, Y., Estrin, Y., Kubin, L., 1996. Statistical Behaviour and Strain Localization Patterns in the Portevin-Le Chatelier Effect. Acta mater. 44.

Lebyodkin, M., Dunin-Barkowskii, L., Brechet, Y., Kubin, L., 2000. Spatio-Temporal Dynamics of the Portevin-Le Chatelier Effect: Experiment and Modelling. Acta mater. 48, 2529-2541.

Lebyodkin, M., Dunin-Barkowskii, L., Lebedkina, T., 2001. Statistical and Multifractal Analysis of Collective Dislocation Processes in the Portevin-Le Chatelier Effect. Physical Mesomechanics 4, 9-14.

Lebyodkin, M., Lebedkina, T., 2008. Multifractal and randomness in the unstable plastic flow near the lower strain rate boundary of instability. Physical Review E 77, 026111-1-026111-8.

Lee, E., 1969. Elastic-plastic deformation at finite strains. J. Appl. Mech. 36, 1-6.

Ling, C., McCormick, P., 1990. Strain Rate Sensitivity and Transient Behaviour in an Al-Mg-Si Alloy. Acta metall. mater 38 (12).

Ling, C., McCormick, P., 1993. The Effect of Temperature on Strain Rate Sensitivity in an Al-Mg-Si alloy. Acta metall. mater. 41, 3127-3131.

Lüders, W., 1860. Über die Äusserung der Elasticität an stahlartigen Eisensta“ ben und Stahlstäben, und über eine beim Biegen solcher Stäbe beobachtete Molecularbewegung. Dingler's Polytechnisches J. 5, 18-22.

Mandel, J., 1974. Thermodynamics and plasticity. In: Delgado Domingos, J., Nina, M., Whitlaw, J. (Eds.), Proc. Int. Symp. Foundations of Continuum Thermodynamics, McMillan, London. 
McCormick, P., 1988. Theory of Flow Localisation due to Dynamic Strain Ageing. Acta metall. 36 (12), 3061-3067.

McCormick, P., Ling, C., 1995. Numerical Modelling of the Portevin-Le-Chatelier Effect. Acta metall. mater. 43 (5), 1969-1977.

McCormick, P., Venkadesan, S., Ling, C., 1993. Propagative Instabilities. An Experimental View. Scripta metall. mater. 29, 1159-1164.

Mesarovic, S. D., 1995. Dynamic Strain Aging and Plastic Instabilities. J. Mech. Phys. Solids 43 (671-700), 5.

Neuhäuser, H., 1990. Plastic instabilities and the deformatino of metals. In: Walgraef, D., Ghoniem, N. M. (Eds.), Patterns, Defects and Material Instabilities.

Penning, P., 1972. Mathematics of the Portevin-Le Chatelier effect. Acta metall. 20, 1169-1175.

Pink, E., Greenberg, A., 1982. Stress drops in serrated flow curves of Al5Mg. Acta Metallurgica 30, 2153-2160.

Portevin, A., Le Châtelier, F., 1923. Sur un phénomène observé lors de l'essai de traction d'alliages en cours de transformation. Comptes Rendus de l'Académie des Sciences, Paris 176, 507-510.

Rizzi, E., Hähner, P., 2004. On the Portevin-Le Châtelier effect: theoretical modeling and numerical results. International Journal of Plasticity 20, 121-165.

Schlipf, J., 1994. On the kinetics of static and dynamic strain aging. Acta Metallurgica and Materialia 31, 909-914.

Simo, J., Miehe, C., 1992. Associative coupled thermoplasticity at finite stains: Formulation, numerical analyses and implementation. Computer Methods in Applied Mechanics and Engineering 98, 41-104.

Sleeswyk, A., 1958. Slow strain hardening of ingot iron. Acta Metallurgica 6, 598-603.

Springer, F., Schwink, C., 1995. On a method to determine directly the waiting time of arrested dislocations and the elementary strain in dynamic strain aging. Scripta Metallurgica et Materialia 32 (11), 1771-1776.

Valanis, K., 2000. Gradient field theory of material instabilities. Arch. Mech. 52 (4-5), 817-838. 
van den Beukel, A., Kocks, U., 1982. The strain dependence of static and dynamic strain aging. Acta Metallurgica 30, 1027-1034.

van den Brink, S., van den Beukel, A., McCormick, P., 1977. The Influence of Specimen Dimensions and Machine Stiffness on the Portevin-Le Chatelier Effect in Al-Cu Alloy. phys. stat. sol. (a) 41.

Zaiser, M., Hähner, P., 1997. Oscilatory modes of plastic deformation:theoretical concepts. Physica Status Solidi (b) 199, 267-330.

Zhang, S., McCormick, P., Estrin, Y., 2001. The Morphology of Portevin-Le Chatelier bands: Finite Element Simulation for Al-Mg-Si. Acta mater. 49, 1087-1094.

Ziegenbein, A., Hähner, P., Neuhäuser, H., 2001. Propagating portevin-le chatelier deformation bands in cu-15 at. al polycrystals: experiments and theoretical description. Materials Science and Engineering A309-310, 336-339. 


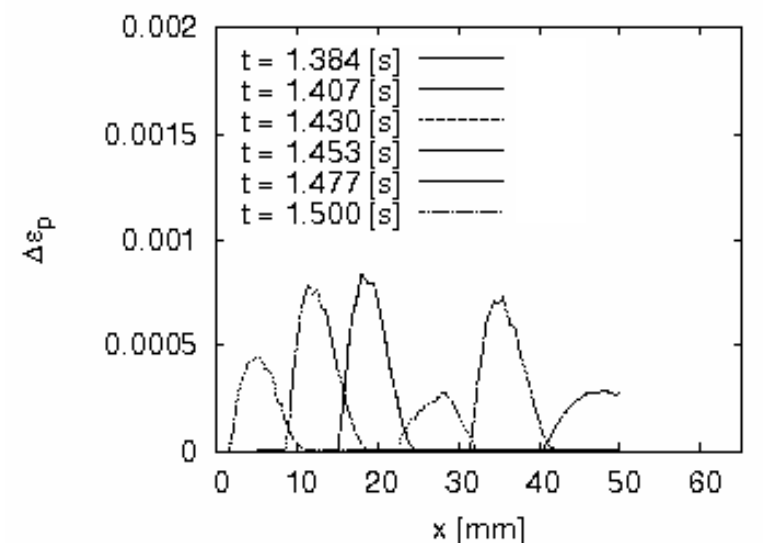

a)

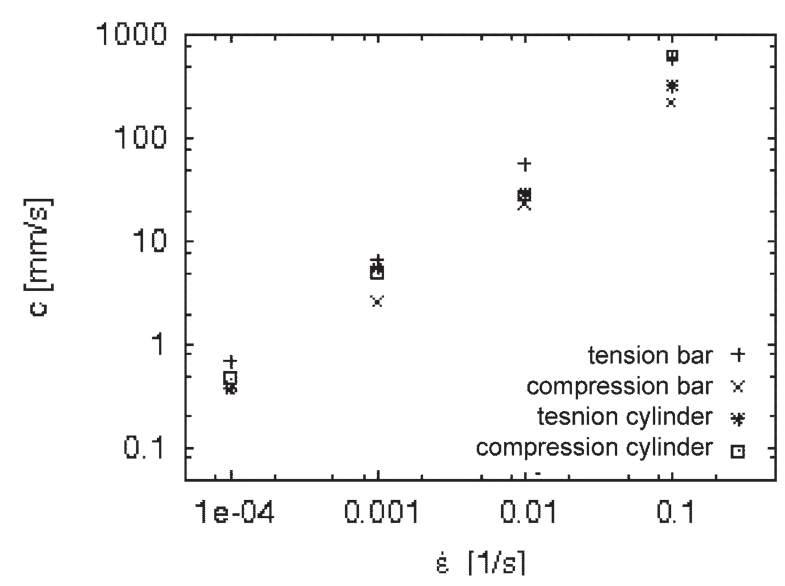

b)

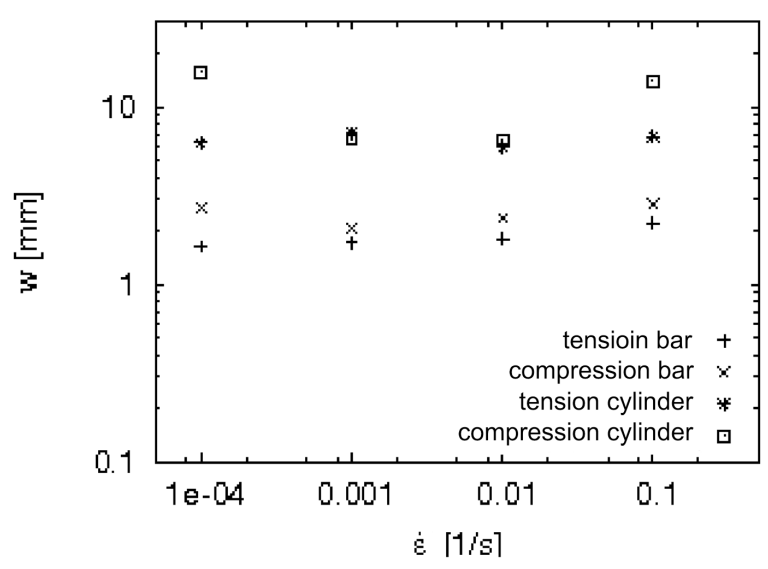

c)

Figure 8: Band characteristics obtained from the simulations: a) distribution of the equivalent plastic strain increment; b) estimated band velocity; and c) estimated band width. The simulation results are obtained for samples with flat and cylindrical geometry subjected to uniaxial tension/compression. 

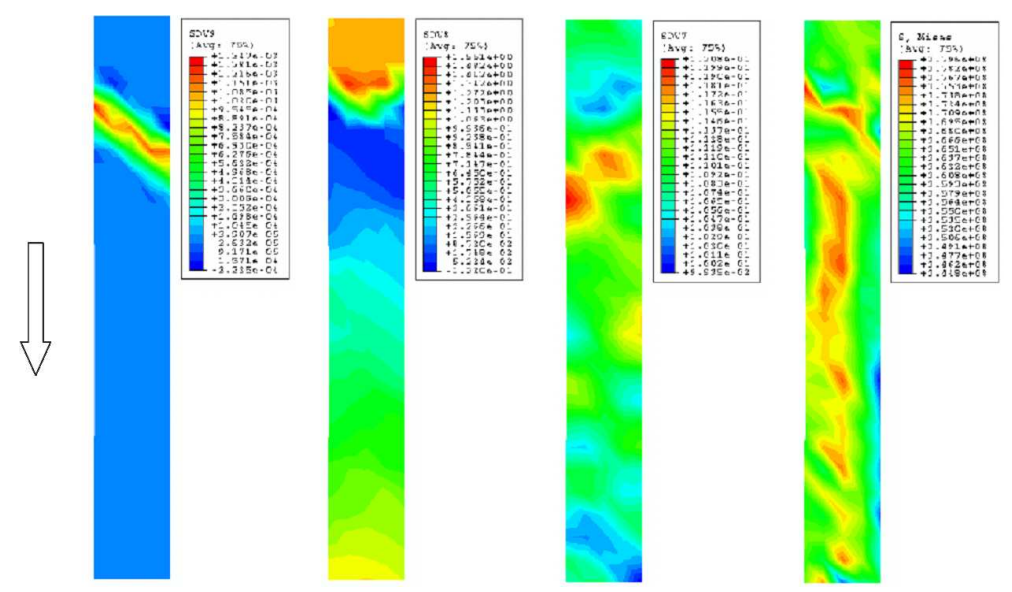

a)
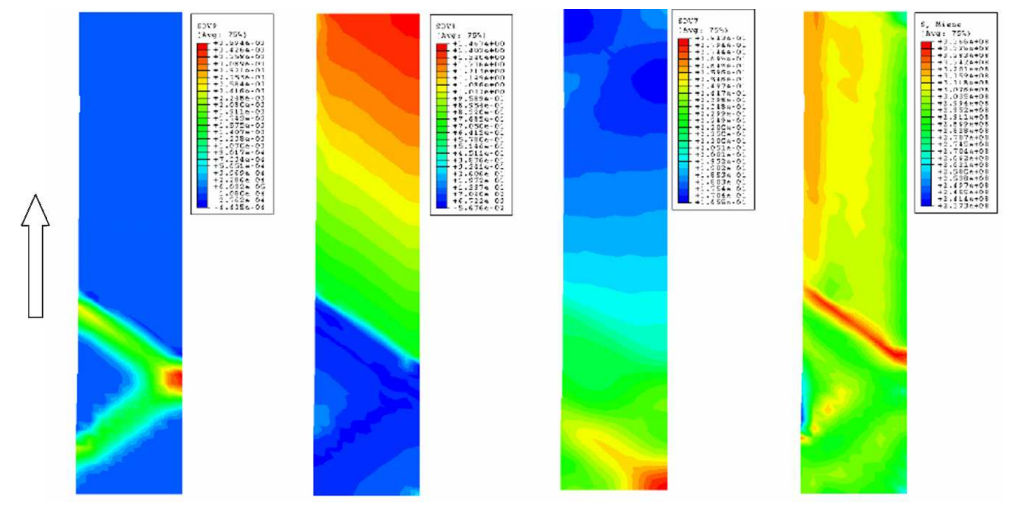

b)

Figure 9: Internal variables and von Mises stress for flat samples subjected to: a) tension; and b) compression. The arrows next to figures indicate the direction of propagation of PLC bands. 


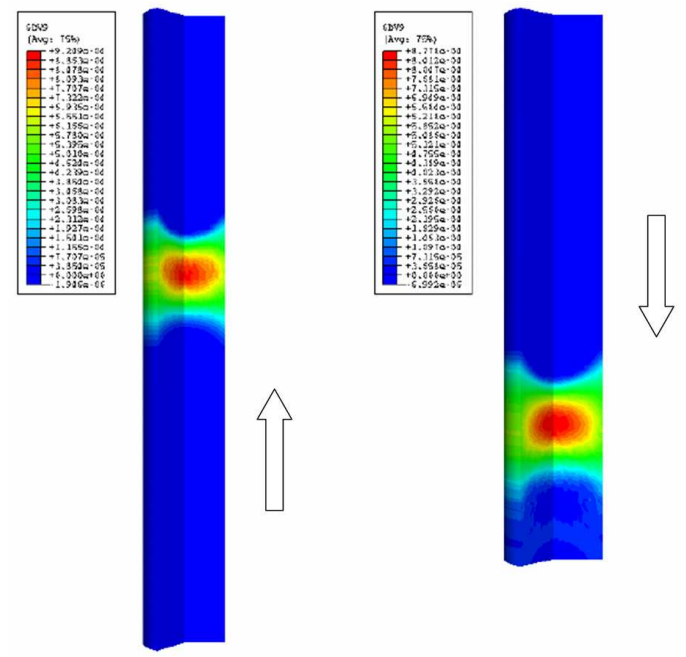

Figure 10: Tension and compression test for cylindrical specimen. The white arrows next to the figures denote the direction of band propagation.

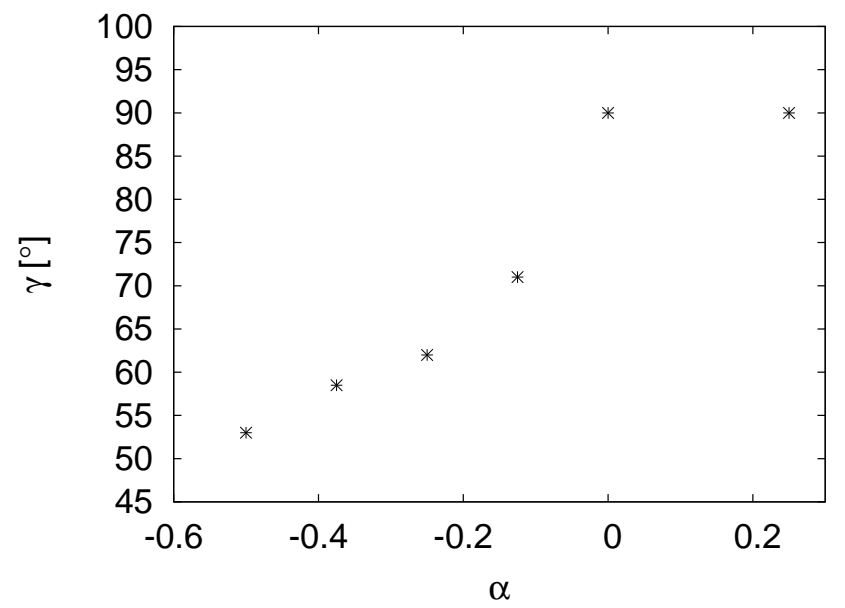

Figure 11: Band orientation angle $\gamma$ (in degrees) as a function of the axiality factor $\alpha$. 


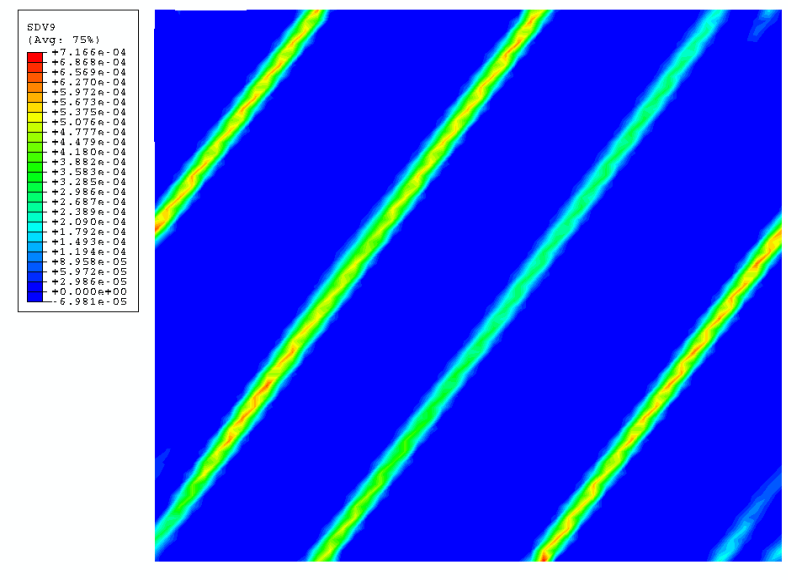

a) $\alpha=-0.375$
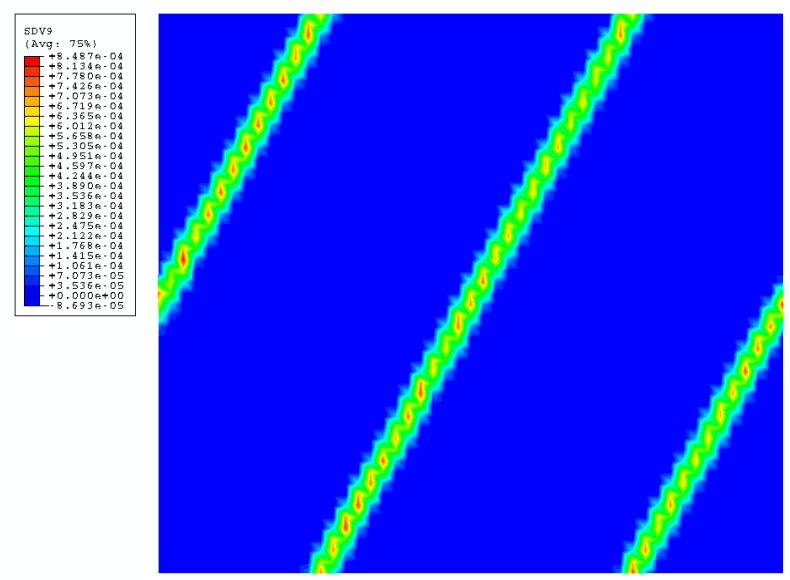

b) $\alpha=-0.25$

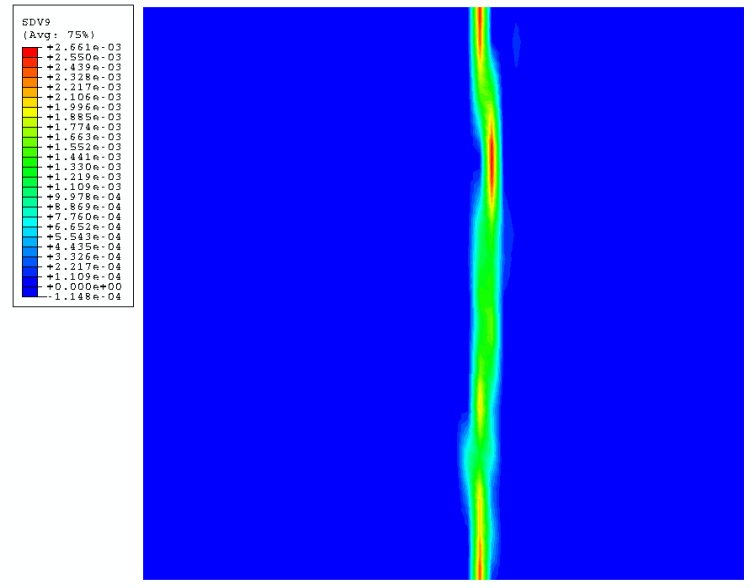

c) $\alpha=0.0$

Figure 12: Calculated distribution of equivalent plastic strain in a 2-dimensional RVE subjected to periodic boundary conditions in the midplane of the metal sheet. Note the change in the band orientation for different strain rates. 

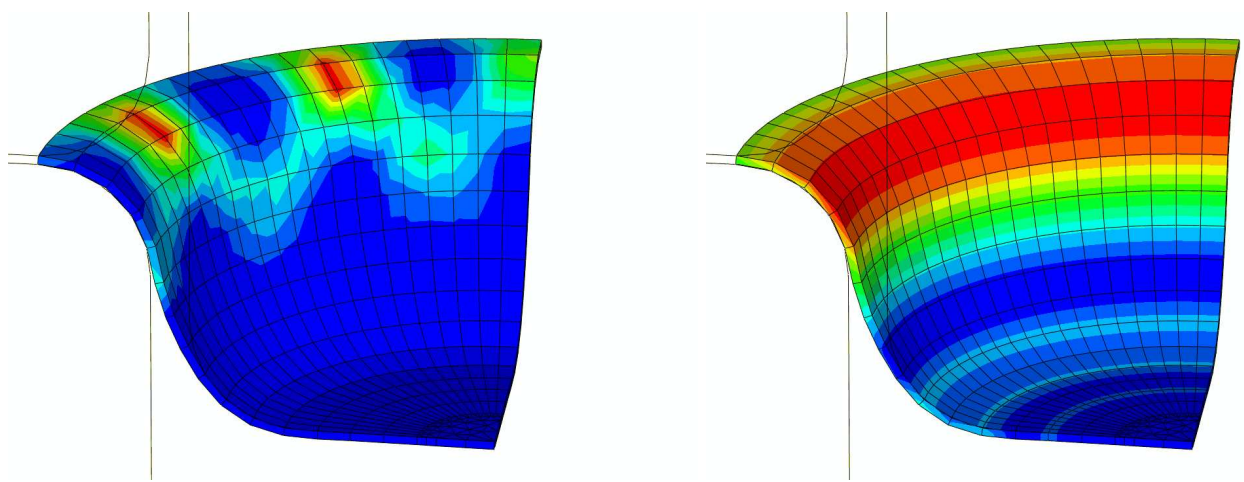

Figure 13: Calculated distribution of equivalent plastic strain increment (left) and accumulated plastic strain (right) during the deep drawing of a circular metal sheet. The PLC effect is observed in circumferential direction in the region with the highest mean curvature, where a high strain concentration (shown in red) is seen. 